\title{
Gestión por Compromisos en el área de almacén de repuestos de una empresa concesionaria automotriz de Lima
}

Cristian Maurizio Segovia Olazábal ${ }^{1}$

\begin{abstract}
RESUMEN
El sector automotriz posee una gran participación en la economía peruana; no obstante, se ha visto afectado en los últimos años por la coyuntura política, variaciones tributarias y la alta competitividad en el sector, lo que ha perjudicado principalmente los resultados comerciales. Ante esta situación, se torna importante la necesidad del desarrollo de una herramienta de gestión que ayude a dirigiry liderar equipos de dicho sector y que esté aplicada a la realidad peruana. El presente estudio se enfocó en el equipo de almacén de repuestos perteneciente a la gerencia de posventa de una empresa concesionaria automotriz en Lima. Asimismo, este estudio expone la implementación de la Gestión por Compromisos como herramienta de gestión y dirección con la finalidad de mejorar los resultados del margen bruto obtenido en la subgerencia a cargo, comprobando su efectividad en los resultados del periodo analizado.
\end{abstract}

Palabras clave: Gestión; compromisos; almacén; repuestos; automotriz.

\section{INTRODUCCIÓN}

En el Perú, el sector automotriz se conforma principalmente por la comercialización de vehículos, carrozados, combustibles, suministros y la oferta de servicio técnico. Negri (2019) señala que el $12 \%$ del PBI nacional es generado por el sector transporte y a su vez produce más de S/ 15000000000 , que equivalen al $14 \%$ de los ingresos tributarios en el Perú. Al respecto, INEI (2020) menciona que el comercio al por menor creció en 3.14\%, impulsado por la venta de combustibles y lubricantes para vehículos automotores, y que el comercio automotriz se incrementó en $4.62 \%$ por la mayor comercialización de vehículos livianos y pesados, lo que derivó en un mayor consumo de autopartes, accesorios, llantas, entre otros; sin embargo, los servicios de mantenimiento preventivo y correctivo mostraron un retroceso en el mismo periodo.

La Asociación Automotriz del Perú (2019) señala que el parque automotor en el Perú, según el Gerente General de la AAP Ellioth Tarazona, posee un índice de motorización de 10.7 -mientras que en Chile, Argentina y México hay entre 3 y 3.3 personas por vehículo-, con una edad de 13.6 años por vehículo, lo cual deriva en un mayor consumo de combustible, contaminación, sobrecostos, entre otros. Además, desde el 2014 se presenta una caída en la importación de vehículos debido al alza del Impuesto Selectivo al Consumo (ISC) vehicular. Cabe precisar, que mediante el Decreto Supremo N. ${ }^{\circ}$ 095-2018-EF (2018), se incrementó entre $20 \%$ a $40 \%$ el impuesto a la adquisición de vehículos; por otro lado, el Decreto Supremo N. ${ }^{\circ} 181-2019-E F$ (2019) modificó nuevamente el ISC reduciendo el impuesto a ciertas cilindradas y elevando el impuesto a la compra y venta de vehículos de segunda mano.

Una de las áreas del sector automotriz que genera un alto porcentaje de los ingresos es la de repuestos, debido a que estos son necesarios para mantener el valor de los bienes. La Asociación Automotriz del Perú (2020) señala que la importación de suministros durante los años 2018 y 2019 presentó una caída

1 Ingeniero industrial por la UNMSM (Lima, Perú). Actualmente, ejerce el cargo de Especialista en Control Logístico y Almacén en Banco Central de Reserva del Perú (Lima, Perú). ORCID: https://orcid.org/0000-0003-3186-4867

E-mail: cristiansegovia@outlook.com 
de $2.6 \%$, lo cual, aunado con el alza de precios y la alta competitividad, repercutió en detrimento del desempeño del sector, pues este enfrenta clientes que buscan mejores precios, lo cual afecta directamente la rentabilidad. En concordancia con lo expresado, se observa la existencia de un potencial decrecimiento en el sector automotriz, así como dificultades por temas tributarios y de competencia, por lo que se hace necesario el desarrollo de una herramienta de gestión que brinde soporte a los equipos interdisciplinarios de dicho sector y que esté adaptada a la realidad peruana.

Dentro del área de repuestos se tiene a las subáreas de ventas, compras y almacén; estas últimas se encargan de los procesos de recepción de mercadería, almacenaje, entrega, codificación y atención de pedidos de taller. Así, este trabajo se centró en el almacén, debido a que los equipos de almaceneros, conformados por auxiliares y encargados, son los responsables de identificar los insumos y piezas requeridas por taller para la reparación o mantenimiento de los vehículos por atender sin descuidar sus labores propias de almacén, por lo que necesitan un sistema de gestión simple que logre impulsar sus actividades y no reduzca el tiempo enfocado en su core-business.

El presente estudio hace uso de la herramienta de Gestión por Compromisos, la cual contribuye a potenciar la productividad de los equipos. Esta herramienta se ha desarrollado y adaptado a la realidad de los concesionarios menores del sector automotriz peruano y busca dar soporte, inicialmente, al área de almacén de repuestos. Esto se debe a que, por su particular casuística, esta área requiere una herramienta de gestión de equipos de almacén que permita canalizar sus actividades diarias en búsqueda del cumplimiento de los objetivos de la gerencia de posventa. Asimismo, el factor más influyente en el corto plazo es el margen bruto del área, debido a que los concesionarios automotrices peruanos poseen un enfoque principalmente comercial, de ahí su elección en este estudio como eje central de los resultados del proyecto.

Cabe señalar que esta herramienta de gestión ha sido adaptada a la realidad de pequeños concesionarios automotrices de la ciudad de Lima a partir de las experiencias obtenidas en la implementación de la Gestión por Compromisos en Divemotor, grupo representante de Mercedes Benz en el Perú. Esta herramienta está enfocada en las gerencias de posventa, por lo que posee un carácter novedoso; además, no es excluyente de otras metodologías existentes. Asimismo, esta metodología puede extenderse a otras áreas de posventa de los concesionarios automotrices, tales como taller, planeamiento de repuestos, ventas de repuestos, entre otros, lo que da cabida a futuras investigaciones para su desarrollo en las áreas comerciales y en otras empresas pertenecientes al sector automotriz.

Según Gestión (2015), el informe de Deloitte "Tendencias Globales de Capital Humano 2015: Liderando en el nuevo mundo del trabajo" muestra que, a nivel mundial, el $87 \%$ de los líderes de recursos humanos y de negocio considera la falta de compromiso como el principal problema de las compañías, siendo en Perú la mayor brecha en "cultura y engagement".

Por otro lado, Repsol (2013) es una compañía con participación en los sectores de petróleo y gas, enfocada en 3 regiones clave: Norteamérica, Latinoamérica y Sudeste Asiático. La compañía busca formar a su personal y otorgarles oportunidades para el desarrollo a través del People Review, donde analizan el potencial y trabajan en sus áreas de mejora. Además, posee dos herramientas de gestión para el reconocimiento laboral: Gestión por Compromisos, donde buscan la cohesión entre los objetivos para el personal operativo, y Gestión por Resultados, para el personal administrativo.

Al respecto, Ramos y Albitres (2010) señalan que el Estado peruano se encuentra en una etapa incipiente en la implementación de Gestión por Resultados, principalmente en eficiencia y calidad del gasto público. Asimismo, identifican deficiencias en capacitación, planificación, programación y capacidad de conducción en detrimento de los objetivos cualitativos y cuantitativos.

Divemotor (2017), distribuidor oficial en Perú de las marcas Mercedes-Benz, Jeep, Dodge, Ram y Freightliner, se encuentra conformado por las empresas Diveimport y Divecenter. La empresa implementó en el 2016 la Gestión por Compromisos (GpC) como herramienta para dirigir los esfuerzos de las áreas comerciales y logísticas hacia la mejora de la rentabilidad de la empresa en un periodo de recesión de inversiones mineras y construcción. Obtuvo como resultado un incremento de $42 \%$ en penetración de mercado en el segmento clientes prospecto, lo que elevó el promedio mensual de ventas en base al desarrollo de clientes. Asimismo, implementó el centro de codificación, que redujo los tiempos de espera de cotizaciones para el canal taller, lo que incrementó la disponibilidad de taller y, por ende, el ticket promedio diario de atención de taller. Además, elevó el indicador "nivel de servicio" de almacén, generado por la atención de 
los pedidos de taller en menor tiempo y con una reducción de errores por reproceso.

En síntesis, estos resultados impactaron directamente en el incremento de la rentabilidad de la empresa a través del incremento del promedio de ventas por taller o por mostrador, originado por mayor ticket de venta por cliente y mayor cantidad de ventas por día debido a la reducción de los tiempos de atención. Cabe señalar que la rentabilidad depende de otros factores como costos y gastos indirectos; el margen bruto, por otra parte, es el factor afectado por las ventas directas.

El marco planteado líneas arriba concluye con el planteamiento del problema principal: ¿Es necesaria la implementación de Gestión por Compromisos en el área de almacén de repuestos para mejorar el margen bruto en una empresa concesionaria del sector automotriz?

El objetivo de esta investigación es determinar la mejora del margen bruto en el área de almacén de repuestos en una empresa concesionaria del sector automotriz con la implementación de gestión por compromisos.

\section{HIPÓTESIS}

En atención a la problemática principal, se plantea la hipótesis "La implementación de Gestión por Compromisos en el área de almacén de repuestos mejora los resultados del margen bruto en la subgerencia de repuestos en una empresa concesionaria del sector automotriz".

- Variable dependiente:

Y1: Margen bruto de la subgerencia de repuestos respecto a ventas totales

- Variable independiente:

X1: Nivel de cumplimiento de gestión por compromisos en el área de almacén de repuestos

Según la Real Academia Española (2020), "compromiso" se define como una obligación contraída, una palabra dada, una dificultad, una promesa. Sin embargo, Sull (2003) —experto en estrategia y ejecución, profesor de estrategia y emprendedurismo en Harvard y London Business School y uno de los 10 nuevos gurús sobre gerencia- señala que un compromiso es una acción tomada en el presente que enlaza una organización a un curso futuro de acción. Es decir, no todas las decisiones gerenciales son compromisos, puesto que dicha acción se convierte en un compromiso si este restringe las futuras alternativas de una empresa en una manera que sería difícil de revertir. Asimismo, sostiene que los compromisos facilitan, en el mediano plazo, la resolución de problemas dentro de las operaciones y organizaciones, y que brindan a los empleados un sentido de enfoque, además de ayudar a priorizar y coordinar acciones.

Por otro lado, Dougherty (2012) —conferencista senior de innovación tecnológica, emprendedurismo y gerencia estratégica de la MIT con experiencia en estabilizar y recapitalizar empresas - sostiene que para que un líder consiga un compromiso emocional con su equipo, necesita comprometerse con ellos también, sin que sea solicitado y sin una expectativa de reciprocidad. También afirma que los compromisos no se pueden forzar o fingir, debido a que esto conlleva a errores y rechazo, por lo que se busca la cohesión con las personas con las que uno trabaja. Por otro lado, señala que los compromisos generan inflexibilidad si son propuestos por largos periodos, por lo que, si el entorno varía, la compañía puede verse atrapada en objetivos desfasados en detrimento de la adaptabilidad, operatividad y competitividad.

\section{Gestión por Compromisos (GpC)}

Dann (2009) sostiene que, según Donald Sull, las jerarquías son sistemas donde las órdenes bajan y la información sube, por lo que no son apropiadas para los trabajos donde se busca la cooperación entre múltiples equipos que poseen diferentes funciones. Tradicionalmente, esta casuística se gestionaba mediante procesos, estandarizaciones y toma de decisiones derivados del enfoque del Total Quality Management (TQM), lo que originaba un alto nivel de estandarización de procesos, pero conllevaba a un menor nivel de innovación. En respuesta, Sull desarrolló el concepto de Gestión por Compromisos, donde esquematiza a una organización como una red de compromisos superpuestos que motivan a los trabajadores a trabajar de manera correcta. Una ventaja es que este concepto sirve para situaciones no estandarizables, tales como estrategias emergentes, innovación, crisis, caos, etc.; además, engloba a toda la supply chain. Al respecto, identifica cinco características que se señalan en la Tabla 1.

Además, Sull y Spinosa (2005) afirman que dicho enfoque no es excluyente de otros, sino que debe tratarse como un complemento para el alcance de mejores resultados, como orientar al personal a alcanzar objetivos y optimizar los procesos productivos. 
Goldsmith (2008) señala que, para incrementar el compromiso en una compañía, los líderes deben generar respeto y confianza, de modo que los colaboradores se sientan ligados a los resultados de dicha organización. Esta técnica incluye la comunicación abierta y efectiva, la transparencia, la verdad, la igualdad, el coaching, el empowerment y el reconocimiento de logros. En la Tabla 2 se esquematizan las recomendaciones para el apoyo de la alta dirección.

\section{Variables generacionales}

Stein y Pin (2009) sostienen que actualmente se vive una integración entre las diferentes generaciones, las cuales conviven en el mismo espacio laboral, lo que impulsa la necesidad de comprender las expectativas, valores, necesidades y motivaciones de la última generación incorporada. El manejo de este punto podría llevar a las empresas a su eventual desarrollo o estancamiento.

Así, los millenials se decantan por empresas donde pueda existir un equilibrio entre su vida personal y laboral, donde tengan acceso a la información, empowerment, comunicación fluida con los líderes de los departamentos, retos y oportunidades de aprendizaje y desarrollo; a lo cual ellos responderán con un alto nivel de preparación, creatividad, iniciativa, entre otras características que ayudarán a los resultados deseados por las compañías.

\section{Motivación y liderazgo}

Newstrom (2011) sostiene que la motivación en el trabajo está compuesta por fuerzas internas y externas que dirigen a un empleado a escoger un curso de acción y actuar de ciertas maneras y que, desde un punto de vista ideal, estas conductas se alinearán al logro de una meta organizacional.

En función a ello, la personalidad y las necesidades de los trabajadores son los principales ejes que los directivos deben evaluar para garantizar la motivación de sus trabajadores. La Figura 1 muestra las principales fuentes de motivación según Newstrom:

Por otra parte, Viato (2014) refiere que el liderazgo consiste en un método para "guiar, acompañar y entrenar a una persona o un grupo con el propósito de alcanzar metas o desarrollar habilidades específicas" (párr. 2). En este proceso, el líder interviene como un entrenador con el fin de que su seguidor mejore el rendimiento de sus funciones centrándolo en la consecución de objetivos.

\section{METODOLOGÍA}

El presente estudio tuvo un carácter cuantitativo basado en un diseño experimental, y la investigación fue de tipo aplicada de nivel explicativo y descriptivo.

Tabla 1. Características de los compromisos.

\begin{tabular}{|c|c|c|c|c|}
\hline \multicolumn{5}{|c|}{ Características de los compromisos } \\
\hline Ser público & Ser activo & Ser voluntario & Ser explícito & Ser motivante \\
\hline
\end{tabular}

Fuente: Dann (2009).

Tabla 2. Tres puntos para generar cultura del compromiso.

\begin{tabular}{|l|l|}
\hline \multirow{2}{*}{$\begin{array}{l}\text { Tres puntos para generar } \\
\text { cultura del compromiso }\end{array}$} & Preparar el ambiente para el diálogo sobre los puntos que se desea abordar. \\
\cline { 2 - 2 } & Expresar mediante números los beneficios por mantener al personal motivado y comprometido. \\
\cline { 2 - 2 } & Obtener aliados y ser colaborativos con ellos. \\
\hline
\end{tabular}

Fuente: Goldsmith (2008).

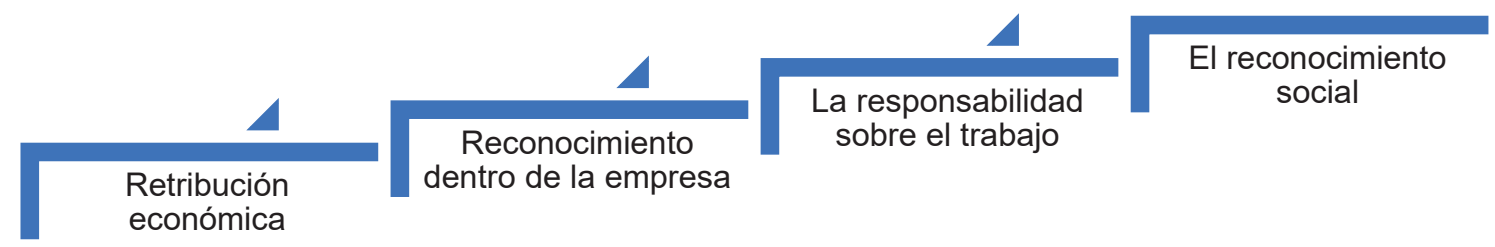

Figura 1. Fuentes de motivación. Fuente: Newstrom (2011). 
La población del estudio está conformada por las 63 concesionarias automotrices que desempeñan sus actividades en Lima y se dedican a la comercialización de vehículos livianos, al servicio de posventa y a la comercialización de repuestos. La muestra es de tipo no probabilístico y, por conveniencia, se definió como muestra a la empresa Limautos Automotriz del Perú S.A.C., debido a que es una empresa que cumple con las características requeridas y se tuvo factibilidad para el desarrollo de la investigación en sus instalaciones.

Las etapas desarrolladas son las siguientes:

\section{Implementación de Gestión por Compromisos}

- Identificar las deficiencias del área en gestión y agruparlas en ejes de mejora o factores según criterios en común.

- Capacitar al personal de almacén y sus jefaturas en Gestión por Compromisos.

- Definir la periodicidad y los intervalos de tiempos que se emplearían para las reuniones de seguimiento de compromisos.

- Diseñar el cuadro de calificación de GpC para la cuantificación de cumplimiento de factores. Dicho cuadro califica con cero o uno cada factor cumplido respecto a la sucursal analizada.

- Iniciar las reuniones de compromisos según los parámetros fijados, en las que, semanalmente, un solicitante establece distintos compromisos y propone una fecha de cumplimiento dirigido a un responsable de ejecución. La semana posterior a cada reunión se llevará a cabo la revisión de los cumplimientos y se deberá dejar constancia de que se ha cumplido con lo solicitado, se ha acordado una nueva fecha o no se ha cumplido.

\section{Evaluación de resultados}

- Generar cada fin de mes un resumen de los efectos de la implementación en el desempeño del personal de almacén con respecto a los factores identificados al inicio del proyecto.

- Evaluar los factores mencionados en cada sucursal y registrarlos en el cuadro de calificación de GpC para la cuantificación de cumplimiento de factores.

- Calcular la calificación mensual del área considerando el promedio obtenido de los factores de las sucursales.
- Diseñar una tabla resumen con los cumplimientos mensuales del área.

- Generar un cuadro con el margen bruto mensual del área.

- Realizar una evaluación de correlación entre el cumplimiento de la Gestión por Compromisos y el desempeño del margen bruto del área en el periodo de ejecución del proyecto.

- A partir de los resultados obtenidos, continuar con la Gestión por Compromisos y realizar los ajustes identificables.

Para los resultados de la variable dependiente, se revisó mensualmente el informe para el directorio, donde se detallan los resultados de ventas, margen bruto por área, inventario, satisfacción de almacén, rentabilidad, entre otros. Con respecto a los resultados de la variable independiente, estos se obtuvieron a partir del cuadro resumen de los cumplimientos mensuales de Gestión por Compromisos. Finalmente, con base en el resultado del proyecto en el plazo coordinado con la gerencia, se determinó el grado de correlación entre la variable independiente y la variable dependiente, a partir del cual se puede afirmar que el proyecto de Gestión por Compromisos aportó mejoras en la productividad del personal de almacén, que se plasmaron en el incremento en el margen bruto de la subgerencia de repuestos, que dio como resultado la validación de la hipótesis planteada.

\section{RESULTADOS}

\section{Implementación de Gestión por Compromisos}

Limautos Automotriz del Perú S.A.C. es una empresa creada en el 2011, que comercializa repuestos de las marcas KIA, Mitsubishi, FUSO, Peugeot, MG y Chery. Posee diez sucursales en Lima, de las cuales, seis sedes brindan atención de repuestos en talleres y cuatro poseen puntos de venta por mostrador. La visión de Limautos es "Ser líder de la industria, manteniendo la capacidad de diferenciación en el mercado e impactando responsable y socialmente en el entorno" (Limautos Automotriz del Perú S.A.C., s.f.). Dicha empresa nunca ha contado con un área de procesos y calidad, por lo que el mapeo de los procesos internos es gestionado por cada gerencia, lo que deriva en que la subgerencia de repuestos, que incluye al área de almacén de repuestos, no cuente con un plan o metodología para gestionar sus resultados y se enfoque en la solución de los problemas diarios relegando a un segundo plano la planificación y gestión de equipos. 
En función de las deficiencias identificadas en la mencionada empresa, se propuso la implementación de GpC como herramienta de gestión para los almacenes de la subgerencia de repuestos a fin de mejorar el margen bruto en dicha subgerencia. Se identificaron los siguientes tres ejes del proyecto, los cuales se planeó gestionar para incrementar el margen bruto de la subgerencia referida:

- El área de almacén no genera ventas directamente; sin embargo, codifica los repuestos requeridos por taller y atiende los repuestos solicitados por mostrador y taller, lo que se tipificaría como ventas indirectas.

- El área de almacén gestiona las existencias de repuestos que puedan generar gastos por ajustes de inventario y gastos por obsolescencia.

- El área de almacén procesa las facturas de los repuestos adquiridos, las cuales, si son ingresadas incorrectamente, pueden generar gastos contables y errores en existencias.

Para dar a conocer el proyecto de la implementación de la Gestión por Compromisos ( $\mathrm{GpC}$ ) al equipo de almacén, se realizó una jornada de capacitación el 27 de diciembre de 2018 a toda la subgerencia de repuestos, en la que se explicó qué son los compromisos; sus estados ("Cumplido", "Pendiente", "No Cumplido" y "Renegociado"); sus implicancias; la facilidad de realizar solicitudes a las jefaturas, y viceversa, para que se comprometan a realizar acciones que faciliten las funciones del día a día; la prioridad del trabajo en equipo, entre otros temas. Adicionalmente, se aplicaron tres dinámicas: Ganar-ganar, Torre de spaguetis y Square Jump, las cuales ayudaron a que el personal de toda la subgerencia se conociera mejor y pudiera desarrollar lazos de amistad. Finalmente, la jornada acabó con un minitorneo de fútbol.

Tomando como base la implementación de Gestión de Compromisos realizada en Divemotor en el 2016, se plantearon reuniones de 20 a 25 minutos los días lunes a las 8:00 a. m. para revisar los compromisos y su cumplimiento. Cabe señalar que la puntualidad es el primer compromiso por desarrollar entre los trabajadores. En la Figura 2 se esquematizan los actos por realizar durante las reuniones por compromisos.

La metodología referida requiere un cuadro de calificación de $\mathrm{GpC}$ para cuantificar el cumplimiento de los factores, por lo que se buscó plantear el mismo esquema usado en Divemotor; sin embargo, por la propia casuística del almacén y su falta de disponibilidad de tiempo, debido a los pendientes generados por el destiempo entre la apertura del taller a las 7:00 a. m. y la apertura de almacén a las 8:00 a. m., se simplificaron los procesos para realizar el seguimiento y se diseñó la Tabla 3 para los almaceneros, el analista de almacén y el subgerente de repuestos.

El cuadro anterior muestra un resultado por cada sucursal que se subdivide en cuatro factores: orden de almacenes, pedidos atendidos a tiempo,

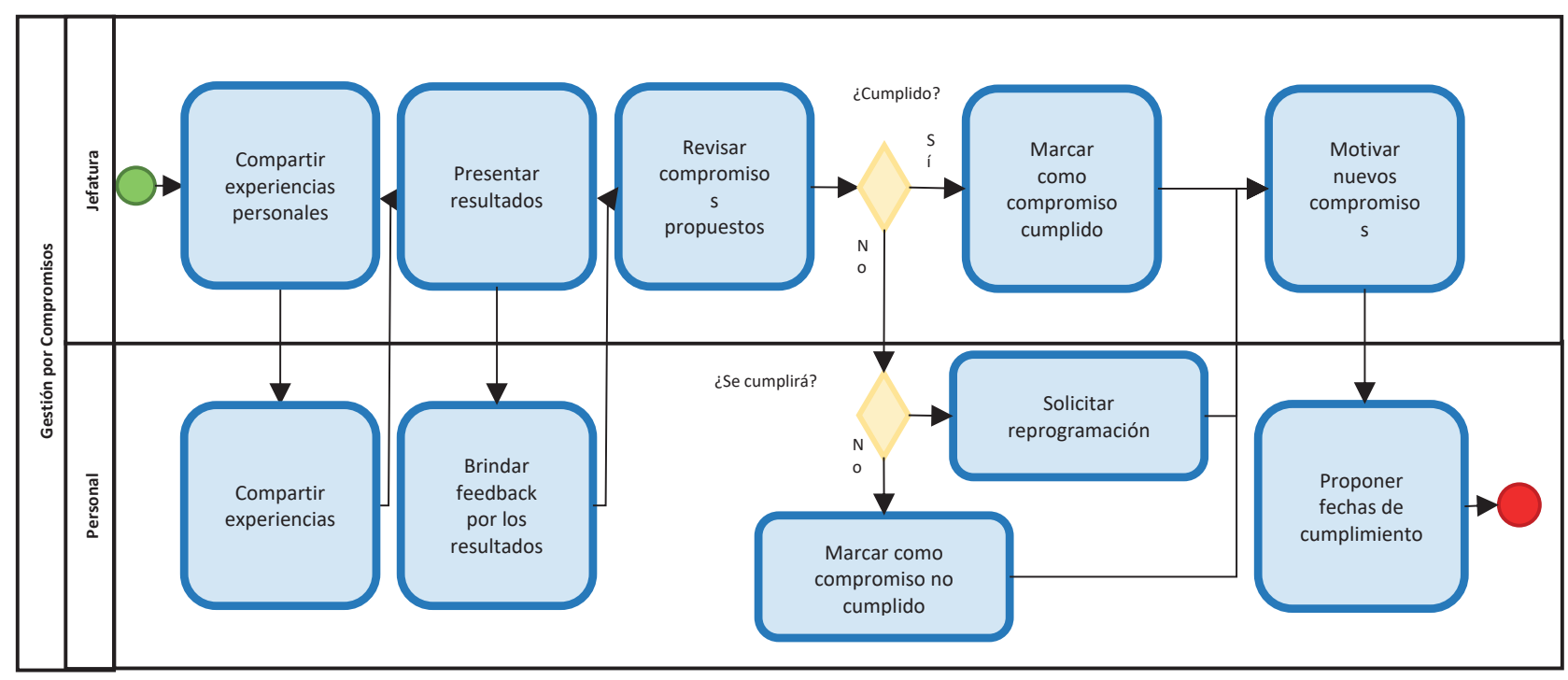

Figura 2. Reuniones por compromisos. Fuente: Elaboración propia. 
Tabla 3. Cuadro de calificación de GpC

\begin{tabular}{|c|c|c|c|c|c|c|c|c|c|c|c|c|c|c|c|c|c|c|c|c|c|c|c|c|c|c|c|c|c|c|c|c|}
\hline & \multicolumn{32}{|c|}{2019} \\
\hline & \multicolumn{4}{|c|}{ Enero } & \multicolumn{4}{|c|}{ Febrero } & \multicolumn{4}{|c|}{ Marzo } & \multicolumn{4}{|c|}{ Abril } & \multicolumn{4}{|c|}{ Mayo } & \multicolumn{4}{|c|}{ Junio } & \multicolumn{4}{|c|}{ Julio } & \multicolumn{4}{|c|}{ Agosto } \\
\hline & 1 & 2 & 3 & 4 & 1 & 2 & 3 & 4 & 1 & 2 & 3 & 4 & 1 & 2 & 3 & 4 & 1 & 2 & 3 & 4 & 1 & 2 & 3 & 4 & 1 & 2 & 3 & 4 & 1 & 2 & 3 & 4 \\
\hline Surquillo1 & & & & & & & & & & & & & & & & & & & & & & & & & & & & & & & & \\
\hline Surquillo2 & & & & & & & & & & & & & & & & & & & & & & & & & & & & & & & & \\
\hline Surquillo3 & & & & & & & & & & & & & & & & & & & & & & & & & & & & & & & & \\
\hline Villarán & & & & & & & & & & & & & & & & & & & & & & & & & & & & & & & & \\
\hline Los Olivos & & & & & & & & & & & & & & & & & & & & & & & & & & & & & & & & \\
\hline Huachipa & & & & & & & & & & & & & & & & & & & & & & & & & & & & & & & & \\
\hline$\% \mathrm{GpC}$ & & & & & & & & & & & & & & & & & & & & & & & & & & & & & & & & \\
\hline
\end{tabular}

Fuente: Elaboración propia.

facturas ingresadas a tiempo y codificación correcta de piezas. A continuación, se detalla que criterios fueron analizados para la evaluación mensual:

a. Orden de almacén: Los almacenes deben estar ordenados, con los ítems codificados y ubicados en los anaqueles correspondientes según sistema. Todo ítem entregado debe ser facturado en el ERP o identificado para pronta facturación. El cumplimiento de este factor genera reducción en gastos de ajustes de inventario.

b. Pedidos atendidos a tiempo: Los picking de los clientes de mostrador y taller deben ser atendidos oportunamente sin errores de cantidades ni de codificación. El cumplimiento de este factor genera una reducción en gastos de ajustes de inventario y un menor tiempo de demora en la atención a clientes de taller, por lo que aumenta la disponibilidad de este, lo cual resulta en un mayor flujo de clientes.

c. Facturas ingresadas a tiempo: Se tiene una meta de 24 horas para los ingresos de facturas y 24 horas adicionales para su envío a la sucursal principal. El cumplimiento de este factor genera una reducción en el tiempo de tareas administrativas y una mayor posibilidad de detectar ítems no conformes dentro de las 24 horas para la presentación de reclamos.

d. Codificación correcta de piezas: Los talleres solicitan diariamente que se codifique, lo cual se debe realizar en el menor tiempo posible según dificultad, disponibilidad de stock, códigos de reemplazo y solicitudes de precio al importador. El cumplimiento de este factor genera una reducción de gastos por obsolescencia y una menor cantidad de reprocesos.
La Tabla 3 fue planificada para ser llenada en una reunión mensual entre el analista de almacén y el coordinador de repuestos y se les dio por cada criterio el valor de uno (1) en caso se haya cumplido o cero (0) si el desempeño del almacén no era el requerido.

El proyecto inició plenamente la primera semana de marzo de 2019. Cada reunión de los lunes fue liderada por el analista de almacén, quien generó los compromisos por sede en función de mejorar sus temas operativos, y se le dio empoderamiento a los almaceneros para que ellos también puedan comprometer al analista y a la subgerencia en acciones que ayuden al mejor desempeño del área. Los compromisos generados durante todo el proyecto fueron detallados en las Tablas 4, 5, 6, 7, 8 y 9.

\section{EVALUACIÓN DE RESULTADOS}

En base a los compromisos gestionados semanalmente, se obtuvo los siguientes resultados a nivel de gestión de almacenes:

Marzo: El personal tuvo un cambio positivo en su horario de llegada debido al compromiso impulsado por la subgerencia. Se corrigieron deficiencias en inventarios de aceites, se identificaron problemas en catálogos por falta de capacitación y se planteó brindar apoyo a sucursales que presentaban alta carga operativa por la entrada de vehículos siniestrados. Se identificaron problemas operativos en Surquillo3 debido a la falta de experiencia del personal asignado, por lo que Surquillo2 brindó inicialmente apoyo. Respecto al ingreso de facturas, dos sucursales acabaron fuera del horario laboral; por 
Tabla 4. Cuadro de compromisos marzo-2019.

\begin{tabular}{|c|c|c|c|c|c|c|c|c|}
\hline \multicolumn{7}{|c|}{ GpC Limautos Repuestos } \\
\hline N. & Fecha & Compromiso & Solicitante & Responsable & Plazo & Cumplimiento & Renegociación & Status \\
\hline 1 & $04 / 03 / 19$ & Puntualidad en el horario de entrada & Subgerente & Todos Almacén & $11 / 03 / 19$ & Renegociado & $18 / 03 / 19$ & Cumplido \\
\hline 2 & $04 / 03 / 19$ & $\begin{array}{c}\text { Realizar el inventario de aceites en } \\
\text { fecha }\end{array}$ & Analista & Todos Almacén & $11 / 03 / 19$ & Renegociado & $18 / 03 / 19$ & Cumplido \\
\hline 3 & $11 / 03 / 19$ & $\begin{array}{c}\text { Solicitar capacitación en catálogo de } \\
\text { repuestos chinos }\end{array}$ & Almacén SQ3 & Analista & $18 / 03 / 19$ & Cumplido & & \\
\hline 4 & $11 / 03 / 19$ & $\begin{array}{r}\text { Enviar personal de apoyo para } \\
\text { ordenar anaqueles }\end{array}$ & Almacén LO & Analista & $18 / 03 / 19$ & Cumplido & & \\
\hline 5 & $11 / 03 / 19$ & $\begin{array}{r}\text { Habilitar apoyo para cotizaciones } \\
\text { grandes de colisión }\end{array}$ & Almacén SQ1 & Analista & $18 / 03 / 19$ & Renegociado & $25 / 03 / 19$ & Cumplido \\
\hline 6 & $18 / 03 / 19$ & $\begin{array}{c}\text { Enviar los sábados fotos de almacén } \\
\text { para evaluación }\end{array}$ & Analista & Todos Almacén & $25 / 03 / 19$ & Cumplido & & \\
\hline 7 & $25 / 03 / 19$ & $\begin{array}{c}\text { Coordinar la llegada a tiempo del } \\
\text { chofer de ruta }\end{array}$ & Almacén LO & Analista & $01 / 04 / 19$ & Cumplido & & \\
\hline 8 & $25 / 03 / 19$ & $\begin{array}{r}\text { Procesar el ingreso de facturas } \\
\text { dentro del cierre }\end{array}$ & Analista & Todos Almacén & $01 / 04 / 19$ & Cumplido & & \\
\hline
\end{tabular}

Fuente: Elaboración propia.

Tabla 5. Cuadro de compromisos abril-2019.

\begin{tabular}{|c|c|c|c|c|c|c|c|c|}
\hline \multicolumn{9}{|c|}{ GpC Limautos Repuestos } \\
\hline $\mathbf{N}^{\circ}$ & Fecha & Compromiso & Solicitante & Responsable & Plazo & Cumplimiento & Renegociación & Status \\
\hline 1 & 01/04/19 & $\begin{array}{l}\text { Reportar repuestos facturados } \\
\text { pendientes de recoger }\end{array}$ & Analista & Todos Almacén & $08 / 04 / 19$ & Cumplido & & \\
\hline 2 & $01 / 04 / 19$ & $\begin{array}{c}\text { Realizar capacitación en seguimien- } \\
\text { to de Back Order }\end{array}$ & Almacén SQ2 & Analista & $08 / 04 / 19$ & Cumplido & & \\
\hline 3 & $08 / 04 / 19$ & Habilitar un catálogo adicional Kia & $\begin{array}{l}\text { Almacén } \\
\text { Villarán }\end{array}$ & Analista & $15 / 04 / 19$ & No Cumplido & & \\
\hline 4 & 08/04/19 & $\begin{array}{c}\text { Habilitar un catálogo adicional } \\
\text { Peugeot }\end{array}$ & Almacén LO & Analista & $15 / 04 / 19$ & Cumplido & & \\
\hline 5 & $15 / 04 / 19$ & $\begin{array}{l}\text { Realizar inventario rotativo de ítems } \\
\text { tipo A para taller }\end{array}$ & Analista & Todos Almacén & $22 / 04 / 19$ & Renegociado & $29 / 04 / 19$ & Cumplido \\
\hline 6 & $15 / 04 / 19$ & $\begin{array}{l}\text { Priorizar devoluciones de repuestos } \\
\text { en plazo }\end{array}$ & Subgerente & Almacén SQ2 & $22 / 04 / 19$ & Cumplido & & \\
\hline 7 & $22 / 04 / 19$ & $\begin{array}{l}\text { Enviar lista de fundas dañadas para } \\
\text { remate }\end{array}$ & Subgerente & Analista & $29 / 04 / 19$ & Renegociado & 06/05/19 & Cumplido \\
\hline 8 & $22 / 04 / 19$ & $\begin{array}{l}\text { Coordinar la fecha de llegada de la } \\
\text { reposición de mayo }\end{array}$ & Almacén SQ2 & Analista & $29 / 04 / 19$ & Cumplido & & \\
\hline 9 & $29 / 04 / 19$ & $\begin{array}{l}\text { Procesar el ingreso de facturas } \\
\text { dentro del cierre }\end{array}$ & Analista & Todos Almacén & $06 / 05 / 19$ & Cumplido & & \\
\hline
\end{tabular}

Fuente: Elaboración propia.

Tabla 6. Cuadro de compromisos mayo-2019.

\begin{tabular}{|c|c|c|c|c|c|c|c|c|}
\hline \multicolumn{7}{|c|}{ GpC Limautos Repuestos } \\
\hline$N^{\circ}$ & Fecha & Compromiso & Solicitante & Responsable & Plazo & Cumplimiento & Renegociación & Status \\
\hline 1 & $06 / 05 / 19$ & $\begin{array}{c}\text { Inventariar mercadería CUP > 2000 } \\
\text { PEN }\end{array}$ & Analista & Todos Almacén & $13 / 05 / 19$ & Renegociado & $20 / 05 / 19$ & Cumplido \\
\hline 2 & $06 / 05 / 19$ & $\begin{array}{c}\text { Apoyar en entrega mercadería Cía. } \\
\text { Seguros }\end{array}$ & Subgerente & Todos Almacén & $13 / 05 / 19$ & Cumplido & & \\
\hline 3 & $06 / 05 / 19$ & $\begin{array}{c}\text { Solicitar capacitación en catálogo } \\
\text { de Peugeot }\end{array}$ & Almacén SQ1 & Analista & $13 / 05 / 19$ & Cumplido & & \\
\hline 4 & $13 / 05 / 19$ & $\begin{array}{c}\text { Cambio de horario de movilidad de } \\
\text { reparto }\end{array}$ & $\begin{array}{c}\text { Almacén } \\
\text { Huachipa }\end{array}$ & Analista & $13 / 05 / 19$ & Cumplido & & No \\
\hline 5 & $13 / 05 / 19$ & $\begin{array}{c}\text { Evaluar rotación de personal du- } \\
\text { rante sábados }\end{array}$ & $\begin{array}{c}\text { Almacén } \\
\text { Villarán }\end{array}$ & Analista & $20 / 05 / 19$ & Renegociado & $27 / 05 / 19$ & Cumplido \\
\hline 6 & $13 / 05 / 19$ & $\begin{array}{c}\text { Incrementar el stock de discos y } \\
\text { pastillas }\end{array}$ & Almacén SQ3 & Analista & $20 / 05 / 19$ & Cumplido & & \\
\hline 7 & $20 / 05 / 19$ & $\begin{array}{c}\text { Programar envío mercadería sin } \\
\text { rotación a Huachipa }\end{array}$ & Almacén LO & Analista & $20 / 05 / 19$ & Cumplido & & \\
\hline 8 & $20 / 05 / 19$ & $\begin{array}{c}\text { Capacitar personal para reemplazo } \\
\text { por vacaciones }\end{array}$ & $\begin{array}{c}\text { Almacén } \\
\text { Huachipa }\end{array}$ & Analista & $20 / 05 / 19$ & Cumplido & & \\
\hline
\end{tabular}


Tabla 6. Continuación...

\begin{tabular}{|c|c|c|c|c|c|c|c|c|}
\hline \multicolumn{1}{|c|}{ GpC Limautos Repuestos } \\
\hline $\mathbf{N}^{\circ}$ & Fecha & Compromiso & Solicitante & Responsable & Plazo & Cumplimiento & Renegociación & Status \\
\hline 9 & $20 / 05 / 19$ & $\begin{array}{c}\text { Habilitar personal adm para apoyo } \\
\text { en recepción }\end{array}$ & Almacén SQ2 & Analista & $27 / 05 / 19$ & Renegociado & $03 / 06 / 19$ & Cumplido \\
\hline 10 & $27 / 05 / 19$ & $\begin{array}{c}\text { Inventariar lubricantes y realizar } \\
\text { balance entre suc. }\end{array}$ & Subgerente & Todos Almacén & $03 / 06 / 19$ & Cumplido & & \\
\hline 11 & $27 / 05 / 19$ & $\begin{array}{c}\text { Procesar el ingreso de facturas } \\
\text { dentro del cierre }\end{array}$ & Analista & Todos Almacén & $03 / 06 / 19$ & Cumplido & & \\
\hline
\end{tabular}

Fuente: Elaboración propia.

Tabla 7. Cuadro de compromisos junio-2019.

\begin{tabular}{|c|c|c|c|c|c|c|c|c|}
\hline \multicolumn{7}{|c|}{ GpC Limautos Repuestos } \\
\hline$N^{\circ}$ & Fecha & Compromiso & Solicitante & Responsable & Plazo & Cumplimiento & Renegociación & Status \\
\hline 1 & $03 / 06 / 19$ & $\begin{array}{c}\text { Solicitar se asigne personal de } \\
\text { limpieza para almacén }\end{array}$ & Almacén SQ3 & Analista & $10 / 06 / 19$ & Cumplido & & \\
\hline 2 & $03 / 06 / 19$ & $\begin{array}{c}\text { Revisar con contabilidad los antici- } \\
\text { pos pendientes }\end{array}$ & Subgerente & Analista & $10 / 06 / 19$ & Cumplido & & \\
\hline 3 & $03 / 06 / 19$ & $\begin{array}{c}\text { Iniciar puntualmente los inventarios } \\
\text { rotativos sabatinos }\end{array}$ & Analista & Todos Almacén & $10 / 06 / 19$ & Cumplido & & \\
\hline 4 & $10 / 06 / 19$ & $\begin{array}{c}\text { Realizar evaluación de objetivos en } \\
\text { plataforma web }\end{array}$ & Subgerente & Todos Almacén & $10 / 06 / 19$ & Cumplido & & \\
\hline 5 & $10 / 06 / 19$ & $\begin{array}{c}\text { Incrementar presupuesto para } \\
\text { alimentos en inv. Rotativ. }\end{array}$ & Analista & Subgerente & $17 / 06 / 19$ & Renegociado & $24 / 06 / 19$ & Cumplido \\
\hline 6 & $17 / 06 / 19$ & $\begin{array}{c}\text { Solicitar al importador mayor rapi- } \\
\text { dez en cotizaciones }\end{array}$ & Almacén SQ1 & Analista & $17 / 06 / 19$ & Cumplido & & \\
\hline 7 & $17 / 06 / 19$ & $\begin{array}{c}\text { Ampliar stock de filtreria y pastillas } \\
\text { para MIT y FUSO }\end{array}$ & Almacén LO & Analista & $17 / 06 / 19$ & Cumplido & & \\
\hline 8 & $24 / 06 / 19$ & $\begin{array}{c}\text { Revisar las devoluciones de rptos } \\
\text { con Pacífico Seg }\end{array}$ & Subgerente & Todos Almacén & $24 / 06 / 19$ & Cumplido & & \\
\hline 9 & $24 / 06 / 19$ & $\begin{array}{c}\text { Coordinar visita con auditoría pre- } \\
\text { via visita fábrica }\end{array}$ & Subgerente & Analista & $24 / 06 / 19$ & Cumplido & & \\
\hline 10 & $24 / 06 / 19$ & $\begin{array}{r}\text { Procesar el ingreso de facturas } \\
\text { dentro del cierre }\end{array}$ & Analista & Todos Almacén & $24 / 06 / 19$ & Cumplido & & \\
\hline
\end{tabular}

Fuente: Elaboración propia.

Tabla 8. Cuadro de compromisos julio-2019.

\begin{tabular}{|c|c|c|c|c|c|c|c|c|}
\hline \multicolumn{9}{|c|}{ GpC Limautos Repuestos } \\
\hline $\mathbf{N}^{\circ}$ & Fecha & Compromiso & Solicitante & Responsable & Plazo & Cumplimiento & Renegociación & Status \\
\hline 1 & 01/07/19 & $\begin{array}{c}\text { Contactar a talleres para atender } \\
\text { rptos separados }\end{array}$ & Subgerente & Todos Almacén & 08/07/19 & Cumplido & & \\
\hline 2 & 01/07/19 & $\begin{array}{c}\text { Enviar sustentos para devolución } \\
\text { de repuestos errados }\end{array}$ & Subgerente & Analista & 08/07/19 & Cumplido & & \\
\hline 3 & 01/07/19 & $\begin{array}{c}\text { Habilitar catálogo de rptos Chery } \\
\text { y MG }\end{array}$ & Almacén SQ3 & Analista & 08/07/19 & Cumplido & & \\
\hline 4 & 08/07/19 & $\begin{array}{c}\text { Comprar material para embalaje - } \\
\text { atención Cia Seg }\end{array}$ & Almacén SQ2 & Analista & 08/07/19 & Cumplido & & \\
\hline 5 & 08/07/19 & $\begin{array}{l}\text { Enviar status destrucción de re- } \\
\text { puestos garantía }\end{array}$ & Analista & Todos Almacén & $15 / 07 / 19$ & Cumplido & & \\
\hline 6 & 08/07/19 & Reducir demora en compras locales & Almacén SQ2 & Analista & $15 / 07 / 19$ & Renegociado & $22 / 07 / 19$ & Cumplido \\
\hline 7 & $15 / 07 / 19$ & $\begin{array}{c}\text { Preparar inventario repuestos por } \\
\text { recoger OT }\end{array}$ & Analista & Todos Almacén & $15 / 07 / 19$ & Cumplido & & \\
\hline 8 & $15 / 07 / 19$ & $\begin{array}{l}\text { Reducir demora en cotizaciones } \\
\text { MG y Chery }\end{array}$ & Almacén SQ3 & Analista & $15 / 07 / 19$ & Renegociado & $22 / 07 / 19$ & Cumplido \\
\hline 9 & $22 / 07 / 19$ & $\begin{array}{c}\text { Revisar repuestos separados por } \\
\text { el sistema }\end{array}$ & $\begin{array}{l}\text { Almacén } \\
\text { Villarán }\end{array}$ & Analista & $22 / 07 / 19$ & Cumplido & & \\
\hline 10 & $29 / 07 / 19$ & $\begin{array}{l}\text { Elevar reclamos por ramales } \\
\text { errados }\end{array}$ & $\begin{array}{c}\text { Almacén LO } \\
\text { - SQ1 } \\
\end{array}$ & Analista & $22 / 07 / 19$ & Cumplido & & \\
\hline 11 & 29/07/19 & $\begin{array}{c}\text { Procesar el ingreso de facturas } \\
\text { dentro del cierre }\end{array}$ & Analista & Todos Almacén & 06/08/19 & Cumplido & & \\
\hline
\end{tabular}

Fuente: Elaboración propia. 
Tabla 9. Cuadro de compromisos agosto-2019.

\begin{tabular}{|c|c|c|c|c|c|c|c|c|}
\hline \multicolumn{9}{|c|}{ GpC Limautos Repuestos } \\
\hline $\mathbf{N}^{\circ}$ & Fecha & Compromiso & Solicitante & Responsable & Plazo & Cumplimiento & Renegociación & Status \\
\hline 1 & $05 / 08 / 19$ & $\begin{array}{c}\text { Actualizar catálogos MG, Mitsubishi } \\
\text { y Chery }\end{array}$ & Almacén LO & Analista & $12 / 08 / 19$ & Cumplido & & \\
\hline 2 & $05 / 08 / 19$ & Habilitar catálogos MIT y FUSO & $\begin{array}{l}\text { Almacén } \\
\text { Huachipa }\end{array}$ & Analista & $12 / 08 / 19$ & Cumplido & & \\
\hline 3 & $12 / 08 / 19$ & $\begin{array}{c}\text { Inventariar mostrador de repuestos } \\
\text { de exhibición }\end{array}$ & Analista & Todos Almacén & $19 / 08 / 19$ & Cumplido & & \\
\hline 4 & $12 / 08 / 19$ & $\begin{array}{c}\text { Gestionar limpieza y orden de zona } \\
\text { de accesorios }\end{array}$ & Subgerente & Analista & $19 / 08 / 19$ & Cumplido & & \\
\hline 5 & $12 / 08 / 19$ & $\begin{array}{l}\text { Capacitar en Excel al personal de } \\
\text { almacén }\end{array}$ & Subgerente & Analista & $19 / 08 / 19$ & Renegociado & 26/08/19 & Cumplido \\
\hline 6 & $19 / 08 / 19$ & $\begin{array}{c}\text { Capacitar en ERP al personal } \\
\text { nuevo }\end{array}$ & $\begin{array}{l}\text { Almacén } \\
\text { SQ3 }\end{array}$ & Analista & $19 / 08 / 19$ & Cumplido & & \\
\hline 7 & $19 / 08 / 19$ & $\begin{array}{l}\text { Reducir tiempo de atención de } \\
\text { Ilantas y baterías }\end{array}$ & $\begin{array}{l}\text { Almacén } \\
\text { SQ2 }\end{array}$ & Analista & 19/08/19 & Cumplido & & \\
\hline 8 & $19 / 08 / 19$ & $\begin{array}{l}\text { Prepararse para visita de fábrica } \\
\text { KIA }\end{array}$ & Subgerente & Todos Almacén & $19 / 08 / 19$ & Cumplido & & \\
\hline 9 & $26 / 08 / 19$ & $\begin{array}{c}\text { Subsanar observaciones por visita } \\
\text { de fábrica KIA }\end{array}$ & Analista & Todos Almacén & $02 / 09 / 19$ & Cumplido & & \\
\hline 10 & $26 / 08 / 19$ & $\begin{array}{l}\text { Procesar el ingreso de facturas } \\
\text { dentro del cierre }\end{array}$ & Analista & Todos Almacén & $02 / 09 / 19$ & Cumplido & & \\
\hline
\end{tabular}

Fuente: Elaboración propia.

lo que, se consideró como "no logrado" en el cuadro de seguimiento de GpC.

Abril: Se brindó capacitación en cuadros de seguimiento para reducir los gastos en obsolescencia, se obtuvieron catálogos adicionales que incrementaron productividad en la codificación de taller, se realizaron inventarios de ítems de alta rotación para reducir las incidencias de faltantes y sobrantes de almacén. En este mes se logró ingresar la mayoría de facturas dentro del horario laboral, lo cual fue resaltado por diferentes gerencias. Aún se encontró la presencia de errores en codificación en sedes con personal nuevo.

Mayo: Se agilizó la entrega de mercadería para mostrador y se obtuvo mayor disponibilidad de almacén, se realizaron capacitaciones por actualizaciones de catálogos, se amplió el nivel de stock de algunas familias de repuestos y se incrementó la recurrencia de inventarios de lubricantes para identificar causales de faltantes de aceites. Respecto al cuadro de seguimiento, se observa un retroceso en el cumplimiento debido a problemas de codificación de siniestros y observaciones en orden de almacén por llegada de repuestos de reposición para stock de 1.5 meses.
Junio: El personal tuvo otro cambio relacionado con la puntualidad en los inventarios sabatinos, lo cual generó que se finalicen en el horario programado; asimismo, se identificaron anticipos pendientes de facturar para disminuir la obsolescencia y se realizaron solicitudes al importador para reducir los tiempos de espera (entre 1 a 3 días) para cotizaciones de ítems de importación. Por otro lado, se gestionaron nuevos parámetros para reposiciones de filtrería debido a la alta variabilidad en el último mes. En general, se obtuvieron los mejores resultados de la gestión por compromisos por la reducción de incidencias.

Julio: En función de reducir la obsolescencia, se realizó el seguimiento a los pendientes de atención de taller. Desde otro punto, se habilitaron nuevos catálogos para repuestos de procedencia china y se dio el inicio a la planificación de destrucción de repuestos de garantía. Nuevamente se solicitó al importador que reduzca el tiempo de respuesta para cotizaciones de repuestos chinos en importación y se iniciaron las gestiones para devolución a fábrica de ramales con errores en codificación por responsabilidad del importador. Adicionalmente, se presentaron dificultades en Surquillo3 por la salida de vacaciones del personal asignado 
y se presentaron errores en codificación de ítems eléctricos de baja rotación.

Agosto: Se realizó una actualización y nueva capacitación de tres catálogos que presentaban errores para la sede de Huachipa. Previo a la visita de fábrica se realizaron inspecciones en cada almacén y se subsanaron temas de orden y limpieza. Como parte de los objetivos anuales, se realizaron capacitaciones en Excel y SPIGA+ para agilizar los procesos internos de almacén. Se coordinó con compras para agilizar las cotizaciones de ítems locales. Para estas fechas, diferentes locales ya consiguieron llegar al $100 \%$ de cumplimiento de GpC, por lo que se solicitó al analista que priorizara las dos sedes que aún poseen dificultades.

En general, se logró puntualidad en los equipos, cumplimientos en fecha de los inventarios rotativos y en el ingreso de facturas. Además, se dio prioridad a las capacitaciones y actualizaciones de catálogos, y se brindó soporte a las diferentes sucursales para jornadas con sobrecarga laboral y cuando se necesitó personal de reemplazo por vacaciones. Finalmente, se cumplió con los cierres de ingresos de facturas a tiempo, acciones que fueron reconocidas por la Gerencia de Administración.
Cabe señalar que durante los meses de enero y febrero se planteó el esquema sin implicar propiamente la gestión por compromisos, la cual se dio por iniciada en su totalidad a partir del mes de marzo de 2019.

Según lo explicado anteriormente, se evaluó cada sucursal en una reunión mensual entre el analista de almacén y el coordinador de repuestos, que generaron los resultados de la Tabla 10.

Se observó un crecimiento en el indicador de cumplimiento de Gestión por Compromisos, el cual inició con $63 \%$ cuando no se aplicaba propiamente el proyecto, y al mes de agosto obtuvo un valor de $88 \%$ : resultado gracias a la cohesión generada en el equipo de almacén.

\section{Contraste de hipótesis: GpC vs Margen Bruto}

A partir de la Tabla 8, se desarrolló la Tabla 11 como resumen, donde se identificó el porcentaje de cumplimiento de $\mathrm{GpC}$ en el periodo del proyecto:

Por otro lado, en la Tabla 12 se señala el porcentaje del margen bruto del área de repuestos desde marzo hasta agosto en el 2019.

Cabe señalar que debido a que el desempeño del canal taller se encontraba bajo el liderazgo del

Tabla 10. Resultados del cuadro de calificación de GpC.

\begin{tabular}{|c|c|c|c|c|c|c|c|c|c|c|c|c|c|c|c|c|c|c|c|c|c|c|c|c|c|c|c|c|c|c|c|c|}
\hline & \multicolumn{32}{|c|}{2019} \\
\hline & \multicolumn{4}{|c|}{ Enero } & \multicolumn{4}{|c|}{ Febrero } & \multicolumn{4}{|c|}{ Marzo } & \multicolumn{4}{|c|}{ Abril } & \multicolumn{4}{|c|}{ Mayo } & \multicolumn{4}{|c|}{ Junio } & \multicolumn{4}{|c|}{ Julio } & \multicolumn{4}{|c|}{ Agosto } \\
\hline & 1 & 2 & 3 & 4 & 1 & 2 & 3 & 4 & 1 & 2 & 3 & 4 & 1 & 2 & 3 & 4 & 1 & 2 & 3 & 4 & 1 & 2 & 3 & 4 & 1 & 2 & 3 & 4 & 1 & 2 & 3 & 4 \\
\hline Surquillo1 & 0 & 1 & 1 & 0 & 0 & 1 & 1 & 0 & 0 & 1 & 1 & 1 & 1 & 1 & 0 & 1 & 1 & 1 & 1 & 0 & 1 & 1 & 1 & 0 & 1 & 1 & 1 & 1 & 1 & 1 & 1 & 1 \\
\hline Surquillo2 & 1 & 0 & 1 & 1 & 0 & 1 & 1 & 0 & 1 & 1 & 1 & 1 & 1 & 1 & 1 & 1 & 0 & 1 & 1 & 1 & 1 & 1 & 1 & 1 & 1 & 1 & 1 & 1 & 1 & 1 & 1 & 1 \\
\hline Surquillo3 & 0 & 1 & 0 & 0 & 1 & 0 & 1 & 0 & 1 & 0 & 0 & 0 & 0 & 1 & 1 & 1 & 0 & 1 & 1 & 0 & 1 & 0 & 1 & 1 & 0 & 1 & 1 & 0 & 1 & 0 & 1 & 0 \\
\hline Villarán & 1 & 1 & 0 & 1 & 1 & 1 & 0 & 1 & 1 & 1 & 0 & 1 & 1 & 1 & 1 & 0 & 1 & 1 & 1 & 0 & 1 & 1 & 1 & 0 & 1 & 1 & 1 & 0 & 1 & 0 & 1 & 1 \\
\hline Los Olivos & 1 & 1 & 1 & 0 & 0 & 1 & 1 & 0 & 1 & 1 & 1 & 0 & 1 & 1 & 1 & 0 & 1 & 1 & 1 & 1 & 1 & 1 & 1 & 1 & 1 & 1 & 1 & 0 & 1 & 1 & 1 & 1 \\
\hline Huachipa & 1 & 1 & 0 & 1 & 1 & 1 & 0 & 1 & 1 & 1 & 1 & 0 & 1 & 1 & 1 & 1 & 1 & 1 & 0 & 1 & 1 & 1 & 1 & 1 & 1 & 1 & 1 & 1 & 1 & 1 & 1 & 1 \\
\hline$\%$ GpC & & 63 & $\%$ & & & 58 & & & & $71^{\circ}$ & & & & 83 & $\%$ & & & 75 & & & & $88^{\circ}$ & $\%$ & & & 83 & $\%$ & & & 88 & $\%$ & \\
\hline
\end{tabular}

Fuente: Elaboración propia.

Tabla 11. Resumen del cuadro de cumplimiento de GpC.

\begin{tabular}{|c|c|}
\hline Mes & GpC \\
\hline MAR & $71 \%$ \\
\hline ABR & $83 \%$ \\
\hline MAY & $75 \%$ \\
\hline JUN & $75 \%$ \\
\hline JUL & $83 \%$ \\
\hline AGO & $88 \%$ \\
\hline
\end{tabular}

Fuente: Elaboración propia. 
Tabla 12. Resultado mensual del Margen Bruto.

\begin{tabular}{|l|c|c|c|c|c|c|c|}
\hline \multicolumn{1}{|c|}{ Margen Bruto } & MAR & ABR & MAY & JUN & JUL & AGO & YTD \\
\hline General & $33 \%$ & $30 \%$ & $28 \%$ & $27 \%$ & $32 \%$ & $30 \%$ & $\mathbf{3 0} \%$ \\
\hline Canal Mostrador & $25 \%$ & $27 \%$ & $19 \%$ & $25 \%$ & $23 \%$ & $29 \%$ & $\mathbf{2 3} \%$ \\
\hline Canal Taller & $37 \%$ & $32 \%$ & $31 \%$ & $30 \%$ & $36 \%$ & $33 \%$ & $33 \%$ \\
\hline
\end{tabular}

Fuente: Elaboración propia.

subgerente de taller, se optó por usar solamente el valor del canal mostrador. Asimismo, en base a lo detallado en la hipótesis, se generó la Tabla 13, en la cual se identificaron los valores para las variables.

Tabla 13. Identificación de variables dependiente e independiente.

\begin{tabular}{|c|c|c|}
\hline Mes & Y: Margen Bruto & X: GpC \\
\hline MAR & $25 \%$ & $71 \%$ \\
\hline ABR & $27 \%$ & $83 \%$ \\
\hline MAY & $19 \%$ & $75 \%$ \\
\hline JUN & $25 \%$ & $75 \%$ \\
\hline JUL & $23 \%$ & $83 \%$ \\
\hline AGO & $29 \%$ & $88 \%$ \\
\hline
\end{tabular}

Fuente: Elaboración propia.

Finalmente, se generó la Tabla 14, donde se determinó el coeficiente de correlación usando Excel 2019, pestaña Datos, apartado Análisis, herramienta Análisis de datos, opción Regresión.

Tabla 14. Estadísticas de la regresión.

\begin{tabular}{|l|l|}
\hline \multicolumn{2}{|c|}{ Estadísticas de la regresión } \\
\hline Coeficiente de correlación múltiple & 0.52467218 \\
\hline Coeficiente de determinación $\mathrm{R}^{\wedge} 2$ & 0.2752809 \\
\hline $\mathrm{R}^{\wedge} 2$ ajustado & 0.09410112 \\
\hline Error típico & 0.03278719 \\
\hline Observaciones & 6 \\
\hline
\end{tabular}

Fuente: Elaboración propia.

Al realizar la revisión de los resultados estadísticos, se observa que el coeficiente de correlación es de 0.52 lo cual señala que sí existe una correlación positiva entre las variables dependientes e independientes, por lo que se da por válida la hipótesis previamente planteada. Sin embargo, al analizar el coeficiente de determinación R2, se observa un valor de 0.275 , lo que significa que el modelo explica levemente la variabilidad de los datos de respuesta en función a su media, por lo que los datos pueden estar alejados de la gráfica de regresión.
Asimismo, se detalla en el análisis brindado por Excel 2019, donde la ecuación de regresión es la siguiente:

$$
Y=0.025+0.28 X
$$

Esta ecuación señala que existe una pendiente positiva, por lo que se refuerza la afirmación de la correlación positiva entre la variable del nivel de cumplimiento de gestión por compromisos en el área de almacén de repuestos y la variable del margen bruto de la gerencia de repuestos respecto a ventas totales.

\section{DISCUSIÓN}

Con respecto al punto anterior, se confirma que, a mayor cumplimiento de la GpC, se incrementa el margen bruto de la gerencia de repuestos en concordancia con los casos de éxito presentados previamente en las implementaciones de Gestión por Compromisos en las empresas Repsol y Divemotor. Esta última, que pertenece al mismo sector empresarial, logró incrementar el promedio mensual de ventas por taller y mostrador, elevando a la par su margen bruto; al igual que la presente empresa donde se desarrolló esta investigación.

Por otro lado, al revisar la tabla de cumplimiento de $\mathrm{GpC}$, se observa un incremento en el cumplimiento de $\mathrm{GpC}$ desde el mes de marzo, fecha en el que se da por válida la capacitación de GpC para el área referida. Además, se observa meses donde hay una caída en el cumplimiento, por lo que se prevé la presencia de factores internos que generaron retroceso en el proyecto en mención durante algunos meses.

Asimismo, luego del análisis realizado, se observó un patrón para el desarrollo de GpC entre colaboradores y jefaturas, lo que da cabida a evaluar la aplicación de esta herramienta en organizaciones que posean una estructura de puestos similar a la planteada en la presente investigación y que dispongan de personal operativo con un nivel de formación secundaria o técnica, para lo cual se recomienda los siguientes pasos a seguir: 
1. Identificar los factores críticos que requieren seguimiento.

2. Solicitar autorización a la gerencia correspondiente sustentando el proyecto en una mejora del margen bruto.

3. Llevar a cabo una reunión inicial de capacitación de GpC para desarrollo de lazos entre los diferentes colaboradores. Asimismo, priorizar la mentalidad ganar - ganar como eje inicial de los compromisos.

4. Generar tablero de seguimiento con los indicadores de los factores identificados.

5. Nombrar personal de seguimiento y elaborar calendario de reuniones.

6. Iniciar las reuniones según la metodología planteada. En las reuniones se debe buscar que la mayoría del equipo participe para incrementar la confianza del personal. Los compromisos deben ser alcanzables y se deben desarrollar con responsabilidad por ambas partes.

7. Cumplidos los seis primeros meses del proyecto, realizar la primera evaluación del margen bruto para reconocer resultados y realizar los ajustes necesarios para brindar continuidad al proyecto.

Adicionalmente, se plantea iniciar en un área End to End para reducir la presencia de factores externos que pueda complicar el cumplimiento de compromisos. De igual manera, se propone la extrapolación a otras empresas que posean una estructura enfocada en ventas y no posean un área de procesos y calidad, como por ejemplo lubricentros, talleres mecánicos, distribuidoras de repuestos, comercializadoras de accesorios entre otras empresas del rubro automotriz.

\section{CONCLUSIONES Y RECOMENDACIONES}

El presente estudio concluye que sí existe una correlación positiva entre el nivel de cumplimiento de gestión por compromisos y el margen bruto de la subgerencia de repuestos enfocado en mostrador, lo cual viene sustentado en el crecimiento del margen durante la ejecución del proyecto planteado.

La Gestión por Compromisos sirve como herramienta para facilitar el trabajo grupal, alinear los esfuerzos en búsqueda de objetivos en común, brindar empowerment al personal operativo y colaborar con el desarrollo de las nuevas generaciones que ingresan al mercado laboral y poseen diferentes motivaciones y metas.

Se recomienda aplicar el modelo presentado durante un plazo mayor para obtener más información y datos numéricos que permitan realizar un análisis completo para la variabilidad de datos respecto a la regresión señalada. Igualmente, debe aplicarse la metodología presentada a la subgerencia de taller en el caso presentado para obtener un panorama completo de la influencia de la Gestión por Compromisos en la Subgerencia de Repuestos en la empresa de muestra.

\section{AGRADECIMIENTOS}

- Universidad Nacional Mayor de San Marcos

- Subgerencia de repuestos de Limautos

\section{REFERENCIAS BIBLIOGRÁFICAS}

[1] Asociación Automotriz del Perú. (2019). AAP: los efectos de un parque automotor escaso $y$ antiguo. Recuperado de https://aap.org. pe/aap-los-efectos-de-un-parque-automotorescaso-y-antiguo-2/

[2] Asociación Automotriz del Perú. (2020). Importación de suministros 2018. Recuperado de https://aap.org.pe/estadisticas/importacion_ suministros/importacion-de-suministros-2019/

[3] Dann, J. (2009). Manage by commitments, not hierarchies by Donald Sull. CBS NEWS. Recuperado de https://www.cbsnews.com/ news/donald-sull-manage-by-commitmentsnot-hierarchies/

[4] D. S. N. ${ }^{\circ}$ 095-2018-EF. Modifican el Literal A del Nuevo Apéndice IV del Texto Único Ordenado de la Ley del Impuesto General a las Ventas e Impuesto Selectivo al Consumo. Diario Oficial El Peruano (miércoles 9 de mayo de 2018).

[5] D. S. N. ${ }^{\circ} 181-2019-E F$. Decreto Supremo que modifica el Impuesto Selectivo al Consumo aplicable a los bienes del Nuevo Apéndice IV del TUO de la Ley del Impuesto General a las Ventas e Impuesto Selectivo al Consumo y el Reglamento de la Ley del Impuesto a la Renta. Diario Oficial El Peruano (sábado 15 de junio de 2019).

[6] Divemotor. (2017). La Empresa. Recuperado de https://www.divemotor.com/empresa/

[7] Dougherty, J. (2012). To get a commitment, make a commitment. Harvard Business Review. 
Recuperado de https://hbr.org/2012/12/to-geta-commitment-make-a-com

[8] Gestión. (2015). El 87\% de empresas considera que la falta de compromiso laboral es su principal problema. Recuperado de https://gestion.pe/tendencias/managementempleo/87-empresas-considera-faltacompromiso-laboral-principal-problema105592-noticia/?ref=gesr

[9] Goldsmith, M. (2008). How to increase Employee Commitment. Recuperado de https:// hbr.org/2008/01/how-to-increase-employeecommi

[10] INEI. (2020). Actividad comercial aumentó $3,47 \%$ en noviembre de 2019. Indicadores Económicos, Boletín Estadístico 02. Recuperado de https://www.inei.gob.pe/ media/MenuRecursivo/boletines/boletinquincenal-02_2.pdf

[11] Limautos Automotriz del Perú S.A.C. (s.f.). Misión. Recuperado de https://www.limautos. pe/nosotros

[12] Negri, A. (2019). 2020: Una meta en común. Asociación Automotriz del Perú, Boletín 125.

[13] Newstrom, J.W. (2011). Comportamiento humano en el trabajo. México D.F., México: McGraw-Hill.

[14] Ramos, L., y Albitres, R. (2010). Sistema de gestión para resultados en el Perú. (Tesis de maestría). Universidad Nacional de Ingeniería, Lima.
[15] Real Academia Española (s.f.). Compromiso. En Diccionario de la lengua española. Recuperado de http://dle.rae.es/srv/ search? $=30 \& \mathrm{w}=$ compromiso

[16] Repsol. (2013). Informes 2013. Equipo Repsol. Retención del Talento.

[17] Sull, D. (2003). Managing by commitments. Harvard Business Review. Recuperado de https://hbr.org/2003/06/managing-bycommitments

[18] Sull, D.N., y Spinosa, C. (2005). Using commitments to manage across units. MIT Sloan Management Review, 47 (1), 73-81. Recuperado de https://www.researchgate.net/ publication/40968864

[19] Stein, G., y Pin, J.R. (2009). Como dirigir a las nuevas generaciones de profesionales: motivaciones y valores de la generación $\mathrm{Y}$. Harvard Deusto Business Review, (178). Recuperado de https://www.harvard-deusto. com/como-dirigir-a-las-nuevas-generacionesde-profesionales-motivaciones-y-valores-dela-generacion-y

[20] Viato, R. (12 de enero de 2014). Poderosa herramienta. Revista $D$. Recuperado de https:// www.prensalibre.com/revista-d/coachingliderazgo-recursos-humanos-gestion-detiempo-desarrollo-profesional-0-1062493984/ 


\section{Commitment-based Management in the Spare Parts Warehouse Area of a Car Dealership in Lima}

Cristian Maurizio Segovia Olazábal ${ }^{1}$

\begin{abstract}
The automotive sector is a large player in the Peruvian economy; however, it has been affected in recent years by the political situation, changes in the tax code and high competitiveness in the sector, which has mainly affected commercial results. In this situation, the need to develop a management tool that helps lead teams in this sector and that is applied to the Peruvian reality becomes important. This study focused on the spare parts warehouse team belonging to the after-sales management of a car dealership in Lima. Likewise, the study shows the implementation of Commitmentbased Management as a management and direction tool to improve the gross margin obtained by the submanagement in charge, by verifying its effectiveness in the results of the period analyzed.
\end{abstract}

Keywords: Management; commitments; warehouse; spare parts; automotive.

\section{INTRODUCTION}

The automotive sector in Peru is mainly comprised of the sale of vehicles, box vans, fuel, supplies, and the offer of automotive services. Negri (2019) states that the automotive sector generates $12 \%$ of the national GDP by producing more than S/ 15000000 000 , which is equivalent to $14 \%$ of tax revenues in Peru. In this regard, INEI (2020) informs that retail trade grew $3.14 \%$ boosted by the sale of fuels and lubricants for automotive vehicles. It also mentions that the motor trade grew $4.62 \%$ due to increased sales of light-duty and heavy-duty vehicles, which resulted in an increased demand of auto parts, accessories, wheels, and other items. However, preventive and corrective maintenance services showed a decline in the same period.

The Asociación Automotriz del Perú (2019) points out that the fleet of motor vehicles in Peru, according to the general manager Ellioth Tarazona, has a motorization rate of 10.7 - whereas in Chile, Argentina, and Mexico there are between 3 and 3.3 people per vehicle-, with an average age of 13.6 years per vehicle, which results in higher fuel consumption, pollution, cost overruns, and other issues. In addition, there has been a drop in vehicle importation since 2014 due to the increase of the Selective Consumption Tax (Impuesto Selectivo al Consumo - ISC) for vehicles. It must be noted that the tax on the acquisition of vehicles was increased between $20 \%$ to $40 \%$ according to the Decreto Supremo N. ${ }^{\circ} 095-2018-E F$ (2018); later, the ISC was modified again through the Decreto Supremo N. ${ }^{\circ} 181-2019-E F$ (2019), in which the tax was reduced for vehicles with certain engine displacements and was raised for the purchase and sale of second-hand vehicles.

One of the areas of the automotive sector that generates a high percentage of revenues is spare parts, as spare parts are necessary to maintain the value of the goods. The Asociación Automotriz del Perú (2020) states that importation of supplies dropped $2.6 \%$ during 2018 and 2019 , which, together with the rise in prices and the high competitiveness, had a detrimental effect on the

1 Industrial Engineer from UNMSM (Lima, Perú). Currently working as Specialist in Logistics Control in Banco Central de Reserva del Perú (Lima, Peru).

ORCID: https://orcid.org/0000-0003-3186-4867

E-mail: cristiansegovia@outlook.com 
sector as it faces customers seeking better prices, thus directly affecting profitability. Accordingly, there is a potential decrease in the automotive sector as well as difficulties due to tax and competition issues, so it is necessary to develop a management tool that supports the interdisciplinary teams in this sector and that is adapted to the situation in Peru.

The spare parts area is comprised by the sales, purchasing and warehouse sub-areas; the latter are responsible for receiving the merchandise, storage, delivery, coding and attending to workshop orders. Therefore, this work was focused on the warehouse area as the teams of warehousemen, made up of assistants and managers, are responsible for identifying the supplies and spare parts required by the workshop for the repair or maintenance of the vehicles to be worked on, without neglecting their own warehouse tasks, so they need a simple management system that manages to boost their activities and does not reduce the time focused on their core business.

This study makes use of the Commitment-based Management tool, which helps boost team productivity. This tool has been developed and adapted to the Peruvian reality of small car dealerships and seeks to support, initially, the spare parts warehouse area due to its particular casuistry as it requires a warehouse equipment management tool that allows channeling its daily activities in order to fulfill the objectives of the after-sales management. Likewise, the most influential factor in the short term is the gross margin of the area, as Peruvian car dealerships mainly focus on sales; for this reason, it was chosen to be the central axis of the project results in this study.

It should be noted that this management tool has been adapted to the reality of small car dealerships in the city of Lima, based on the experiences obtained in the implementation of Commitment-based Management in Divemotor, the group representing Mercedes Benz in Peru. This tool is focused on after-sales management, so it is of a novel nature that does not exclude other existing methodologies. Likewise, this methodology can be extended to other after-sales areas of car dealerships, such as workshop, spare parts planning, spare parts sales, among others, which gives room for future research about its implementation in commercial areas and in other companies belonging to the automotive sector.

According to Gestión (2015), the Deloitte report Tendencias Globales de Capital Humano 2015: Liderando en el nuevo mundo del trabajo shows that $87 \%$ of human resources and business leaders worldwide consider the lack of engagement as the main concern of companies. The biggest gap in Peru would be "culture and engagement".

On the other hand, Repsol (2013) is an oil and gas sector company focused on 3 key regions: North America, Latin America, and Southeast Asia. The company seeks to train its personnel and provide them with opportunities for development through performance review, where they analyze their potential and work on their areas of improvement. It also has two management tools for employee recognition: Commitment-based Management, where they seek cohesion between objectives for operational personnel, and Results-based Management, for administrative personnel.

In this regard, Ramos and Albitres (2010) point out that the Peru is still in its infancy regarding the implementation of Results-based Management, mainly in efficiency and quality of public spending. They also identify deficiencies in training, planning, programming, and management capacity to the detriment of qualitative and quantitative objectives.

Divemotor (2017), official distributor in Peru of Mercedes-Benz, Jeep, Dodge, Ram, and Freightliner, is comprised of the companies Diveimport and Divecenter. In 2016, the company implemented Commitment-based Management (CbM) as a tool to direct the efforts of the commercial and logistics areas towards improving the profitability of the company within a period of recession in mining and construction investments. This resulted in a $42 \%$ increase in market penetration in the prospective client segment, which increased the average monthly sales based on client development. It also implemented a coding center, which reduced waiting times for quotations for the workshop channel, increasing workshop availability and, therefore, the average daily workshop service. In addition, it increased the "service level" indicator of the warehouse because of the faster service of workshop orders and reduced in manufacturing reworks.

In summary, these results had a direct impact on the profitability increase of the company due to the raise in the average sales per workshop or per counter, originated by a higher average monthly sales per customer and a greater number of sales per day as a result of the reduction in service times. It should be noted that profitability depends on factors such as overhead costs and expenses; gross margin, on the other hand, is the factor affected by direct sales. 
The framework outlined above concludes with the statement of the main problem: Is the implementation of Commitment-based Management in the spare parts warehouse area necessary to improve the gross margin in a car dealership?

The objective of this research is to determine the improvement of the gross margin in the spare parts warehouse area of a car dealership after the implementation of Commitment-based management.

\section{HYPOTHESIS}

In response to the main problem, the hypothesis proposed is "The implementation of Commitment-based Management in the spare parts warehouse area improves the results of the gross margin in the spare parts sub-management in a car dealership".

\section{- Dependent variable:}

Y1: Gross margin of the spare parts sub-management with respect to total sales

- Independent variable:

X1: Compliance level of Commitment-based Management in the spare parts warehouse area

According to Real Academia Española (2020), "commitment" is defined as an obligation undertaken, a word given, a difficulty, a promise. However, Sull (2003) -an expert in strategy and execution, professor of strategy and entrepreneurship at Harvard and London Business School and one of the 10 new management gurus - points out that a commitment is an action taken in the present that binds an organization to a future course of action. In other words, not all management decisions are commitments, since such decisions become a commitment if it constrains future alternatives a company in a way that would be difficult to reverse. He also argues that, in the medium term, commitments facilitate the resolution of problems within operations and organizations, and that they provide employees with a sense of focus, as well as helping to prioritize and coordinate actions.

On the other hand, Dougherty (2012) —a senior lecturer in technological innovation, entrepreneurship, and strategic management at MIT with experience in stabilizing and recapitalizing companies-argues that for a leader to achieve emotional engagement with their team, it is necessary to engage with them as well, unsolicited and without an expectation of reciprocity. He also states that commitments cannot be forced or faked, because this leads to mistakes and rejection, so cohesion is sought with the people with whom one works. On the other hand, he points out that commitments generate inflexibility if they are proposed for long periods, so if there are changes in time, the company may be trapped in outdated objectives to the detriment of adaptability, operability, and competitiveness.

\section{Commitment-based Management (CbM)}

Dann (2009) argues that, according to Donald Sull, hierarchies are systems where orders go down and information goes up, so they are not appropriate for jobs where cooperation between multiple teams with different functions is sought. Traditionally, this casuistry was managed through processes, standardizations and decision making derived from the Total Quality Management (TQM) approach, which resulted in a high level of process standardization, but led to a lower level of innovation. In response, Sull developed the concept of Commitment-based Management, where he schematizes an organization as a network of overlapping commitments that motivate workers to work in the right way. One advantage of this concept is that it can be used in non-standard situations, such as emerging strategies, innovation, crisis, chaos, etc.; it also encompasses the entire supply chain. In this regard, it identifies five characteristics, which are shown in Table 1.

In addition, Sull and Spinosa (2005) state that this approach does not exclude others but should be treated as a complement to achieve better results, such as guiding personnel to achieve objectives and optimizing production processes.

Goldsmith (2008) points out that leaders must inspire respect and trust in order to increase commitment in a company, so that employees feel linked to the results of the organization. This technique includes open and effective communication, transparency, truth, equality, coaching, empowerment, and recognition of achievements. Table 2 outlines the recommendations for top management support.

\section{Generation variables}

Stein and Pin (2009) argue that there is currently an integration between the different generations that coexist in the same workspace. This boosts the need to understand the expectations, values, needs and motivations of the latest generation incorporated. The management of this situation could lead companies to their eventual development or stagnation. 
Millennials opt for companies that offer a balance between their personal life and work, where they have access to information, empowerment, fluid communication with department leaders, challenges and opportunities for learning and development. In return, they will offer a high level of preparation, creativity, initiative, among other characteristics that will help to achieve the results desired by the companies.

\section{Motivation and Leadership}

Newstrom (2011) argues that motivation at work is comprised of internal and external forces that direct an employee to choose a course of action and act in certain ways and that, from an ideal point of view, these behaviors will be directed to the achievement of an organizational goal.

Accordingly, the personality and needs of the employees are the main axes that managers must evaluate to ensure their employees are motivated. Figure 1 shows the main sources of motivation according to Newstrom:

On the other hand, Viato (2014) refers that leadership consists of a method intended to guiar, acompañar y entrenar a una persona o un grupo con el propósito de alcanzar metas o desarrollar habilidades específicas [guide, accompany and train a person or a group of people with the purpose of achieving goals or developing specific skills] (para. 2 ). In this process, the leader intervenes as a coach for his follower to improve the performance of their functions by focusing on the achievement of objectives.

\section{METHODOLOGY}

This study is quantitative based on an experimental design. The type is applied, and the level is explanatory and descriptive.

The study population is made up of 63 car dealerships that operate in Lima and are engaged in the sale of light-duty vehicles, after-sales services, and the commercialization of spare parts. The company Limautos Automotriz del Perú S.A.C. was defined as the sample, which is non-probabilistic, as it is a company that meets the required characteristics and it was feasible to carry out the research at its facilities.

The stages developed are as follows:

\section{Implementation of Commitment-based Management}

- Identify deficiencies in the area and group them into improvement axes or factors according to common criteria.

Table 1. Commitment Characteristics.

\begin{tabular}{|c|c|c|c|c|}
\hline \multicolumn{5}{|c|}{ Commitment Characteristics } \\
\hline Public & Active & Voluntary & Explicit & Motivating \\
\hline
\end{tabular}

Source: Dann (2009).

Table 2. Three Points to Generate a Culture of Commitment.

\begin{tabular}{|l|l|}
\hline \multirow{3}{*}{$\begin{array}{l}\text { Three points to generate } \\
\text { a culture of commitment }\end{array}$} & Prepare atmosphere for dialogue on the points to be addressed. \\
\cline { 2 - 2 } & Express through figures the benefits of keeping staff motivated and engaged. \\
\cline { 2 - 2 } & Obtain allies and be collaborative with them. \\
\hline
\end{tabular}

Source: Goldsmith (2008).

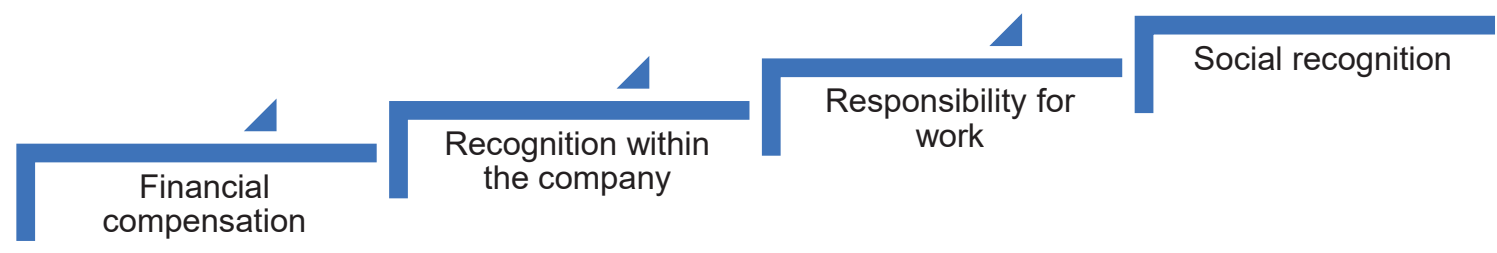

Figure 1. Motivation Source.

Source: Newstrom (2011). 
- Train warehouse personnel and their managers in Commitment-based Management.

- Define the frequency and time intervals to be used for commitment monitoring meetings.

- Design the CbM rating chart for the quantification of factor compliance. This table rates with zero or one each factor fulfilled for each branch analyzed.

- Start the commitment meetings according to the parameters set, in which, on a weekly basis, an applicant establishes different commitments and proposes a date of compliance and a responsible for execution. The week after each meeting, compliance will be reviewed and record will be kept of whether the request has been complied with, a new date has been agreed on or the request has not been fulfilled.

\section{Evaluation of Results}

- Generate a month-end summary of the implementation effects on the performance of the warehouse personnel with respect to the factors identified at the beginning of the project.

- Evaluate the aforementioned factors in each branch and record them in the CbM rating chart to quantify factor compliance.

- Calculate the monthly rating of the area considering the average obtained from the factors from each branch.

- Design a summary chart with the monthly compliance of the area.

- Create a chart containing the monthly gross margin of the area.

- Perform a correlation assessment between the Commitment-based Management and the gross margin performance of the area during the project execution.

- Based on the results obtained, continue with the Commitment-based Management and make the identifiable adjustments.

To obtain the results of the dependent variable, the monthly report to the board of directors was reviewed, where the results of sales, gross margin by area, inventory, warehouse satisfaction, profitability, among others, are detailed. The results of the independent variable were obtained from the summary chart of the monthly fulfillment with the Commitment-based Management. Finally, based on the project results in the term coordinated with management, the degree of correlation between the independent variable and the dependent variable was determined, from which it can be affirmed that the Commitment-based Management project improved the productivity of the warehouse personnel, which was reflected in the increase of the gross margin of the spare parts sub-management, resulting in the validation of the hypothesis proposed.

\section{RESULTS}

\section{Implementation of Commitment-Based Management}

Limautos Automotriz del Perú S.A.C. is a company established in 2011 that sells spare parts for the KIA, Mitsubishi, FUSO, Peugeot, MG, and Chery brands. It has ten branches in Lima, six of which offer spare parts service in workshops and four of which have counter sales points. Its vision is Ser líder de la industria, manteniendo la capacidad de diferenciación en el mercado e impactando responsable y socialmente en el entorno [Be the industry leader, maintaining the capacity for differentiation in the market and have a responsible and social impact on the environment] (Limautos Automotriz del Perú S.A.C., n.d.). This company has never had a process and quality area, so the mapping of internal processes is managed by each management, which means that the spare parts sub-management, which includes the spare parts warehouse area, does not have a plan or methodology to manage its results and focuses on solving daily problems, overshadowing planning and equipment management.

Based on the deficiencies identified in the aforementioned company, the implementation of $\mathrm{CbM}$ was proposed as a management tool for the warehouses of the spare parts sub-management in order to improve its gross margin. The following three axes of the project were identified and their management was planned to increase the gross margin of the spare parts sub-management:

- The warehouse area does not generate sales directly; however, it codes the spare parts required by workshops and attends the spare parts requested by counter and workshop, which would be typified as indirect sales.

- The warehouse area manages the stock of spare parts that may generate expenses for inventory adjustments and expenses due to obsolescence.

- The warehouse area processes invoices for purchased spare parts, which, if entered 
incorrectly, can generate accounting expenses and inventory errors.

In order to introduce the Commitment-based Management $(\mathrm{CbM})$ implementation project to the warehouse team, a training day was held for the spare parts sub-management personnel on December 27, 2018. In this training, it was explained what commitments are, their status ("Fulfilled", "Pending", "Not Fulfilled" and "Renegotiated"), their implications, the ease of making requests to management and vice versa, so that they commit to actions that facilitate day-to-day functions, the priority of teamwork, among other topics. In addition, three group-dynamic games were applied: Win-win, Spaghetti Tower, and Square Jump, which helped the sub-management personnel to get to know each other better and develop bonds of friendship. Finally, the day ended with a soccer tournament.

Based on the Commitment-based Management implemented at Divemotor in 2016, meetings of 20 to 25 minutes were scheduled on Mondays at 8:00 a.m. to review commitments and their fulfillment. It should be noted that punctuality was the first commitment to be developed among workers. Figure 2 shows the actions to be carried out during the commitment meetings.

The methodology selected requires a $\mathrm{CbM}$ rating chart to quantify compliance with the factors, so the same scheme used at Divemotor was used. However, due to the warehouse characteristics and its lack of time availability as a consequence of the backlog generated as a result of the interval between the opening of the workshop at 7:00 a.m. and the opening of the warehouse at 8:00 a.m., the processes were simplified in order to have the meetings at 7:00 a.m. and 8:00 a.m., respectively, and Table 3 was designed for the warehouseman, the warehouse analyst and the spare parts manager.

The above table shows a result per branch, which is subdivided into four factors: warehouse order, orders served on time, invoices entered on time and correct coding of parts. The criteria used for the monthly evaluation are detailed below:

a. Warehouse order: The warehouses must be in order, with the items coded and located on the corresponding shelves according to the system. All items delivered must be invoiced in the ERP system or identified for prompt invoicing. Compliance with this factor results in a reduction in expenses for inventory adjustment.

b. Orders complete on time: Pickings for counter and shop customers must be handled in a timely manner with no errors in quantities or coding. Compliance with this factor reduces the expenses for inventory adjustments and the delay in attending to workshop customers, thus increasing their availability, which results in a greater flow of customers.

c. Invoices entered on time: There is a deadline of 24 hours to receive invoices and an additional 24 hours to send them to the main branch.

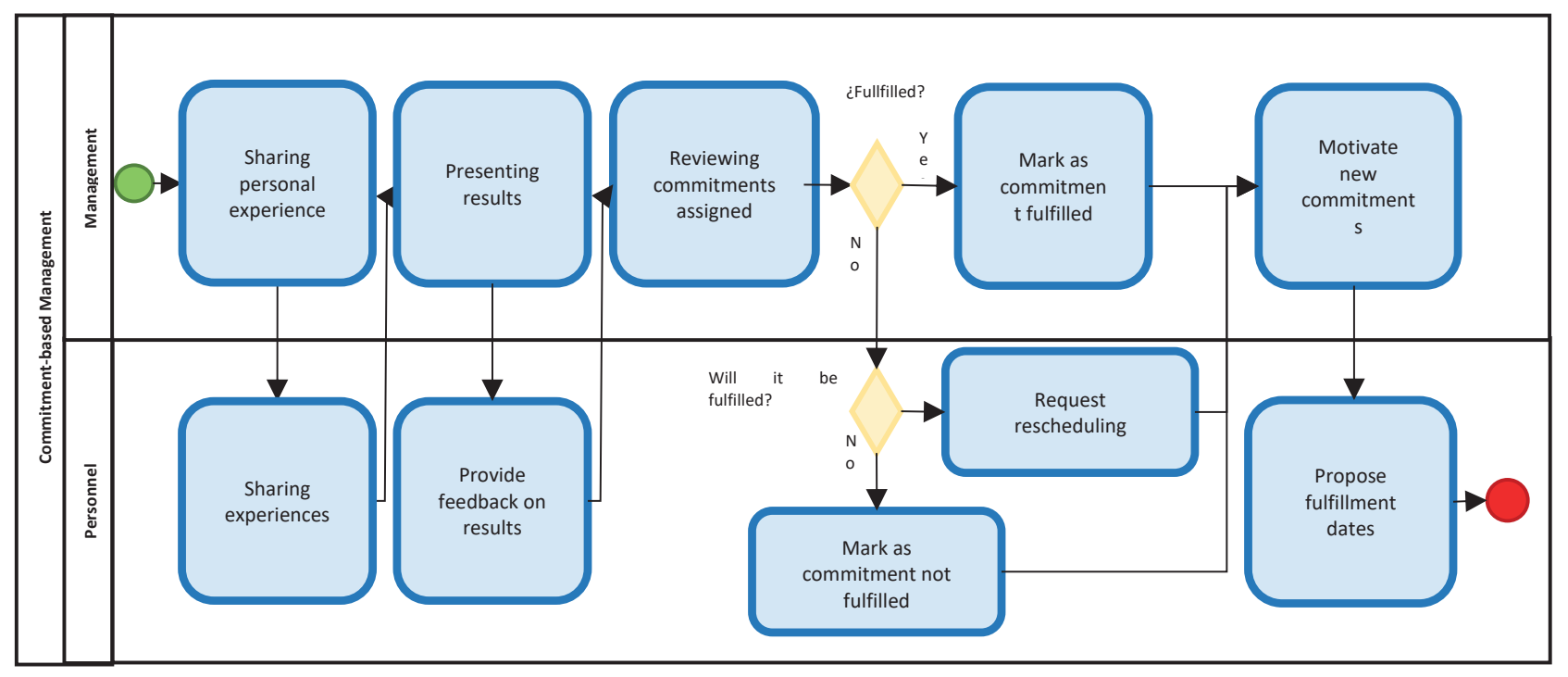

Figure 2. Commitment Meetings.

Source: Prepared by the author. 
Table 3. CbM Rating Chart.

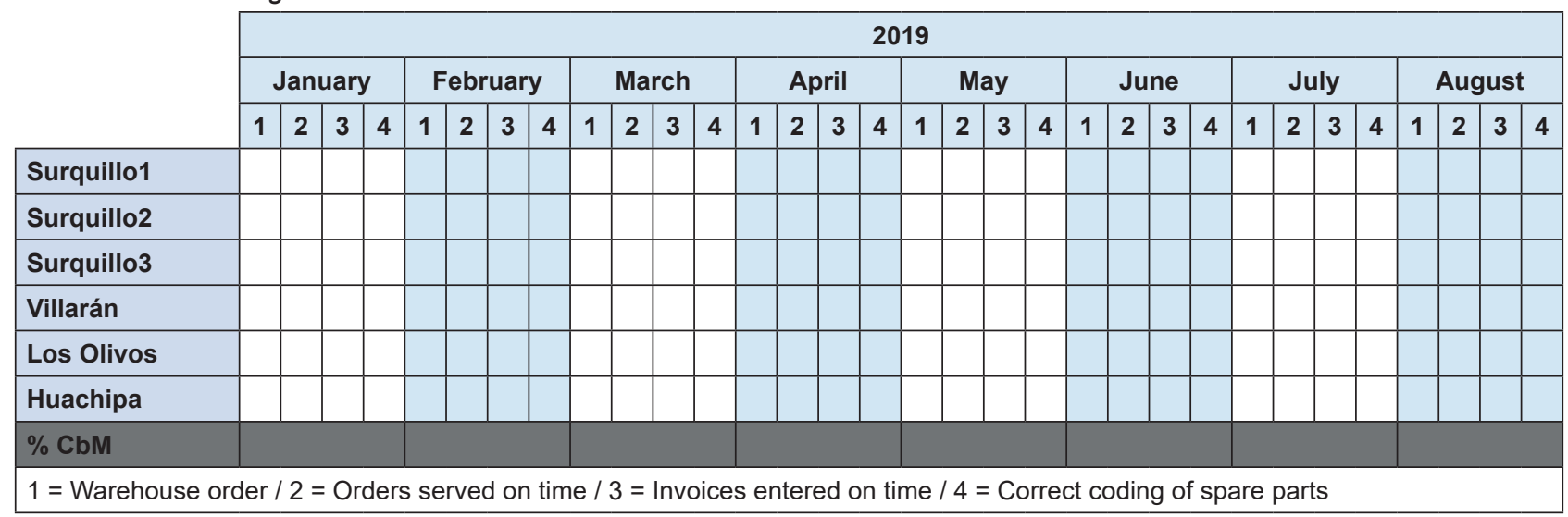

Source: Prepared by the author.

Compliance with this factor reduces administrative task times and offers a greater possibility of detecting nonconforming items within 24 hours for filing claims.

d. Correct coding of spare parts: The workshops request daily coding, which must be done in the shortest possible time according to difficulty, stock availability, replacement codes and price requests to the importer. Compliance with this factor reduces obsolescence costs and results in a lower number of reprocesses.

Table 3 was planned to be filled out in a monthly meeting between the warehouse analyst and the spare parts coordinator. They were given a value for each criterion of one (1) if it was met or zero (0) if the warehouse performance was not as required.

The project started in full the first week of March 2019. Each Monday meeting was led by the warehouse analyst, who proposed the commitments per each branch in order to improve their operational issues, and storekeepers were empowered so they could also engage the analyst and the assistant manager in actions that would help the area perform better. The commitments proposed throughout the project are detailed in Tables $4,5,6,6,7,8$, and 9.

\section{EVALUATION OF RESULTS}

Based on the commitments managed weekly, the following results were obtained at warehouse management level:

March: The personnel had a positive change in their arrival schedule due to the commitment promoted by the sub-management.
Deficiencies in oil inventories were corrected, problems due to lack of training were identified in catalogs, and support was provided to branches that had a high operational load due to the entry of damaged vehicles. Operational problems were identified in Surquillo3 due to the lack of experience of the assigned personnel, for which Surquillo2 initially provided support. Regarding invoice entry, two branches finished out of working hours; therefore, it was considered as "not fulfilled" in the commitment chart.

April: Training was provided on tracking charts to reduce expenses due to obsolescence, additional catalogs were obtained to increase productivity in workshop coding, and inventories of high turnover items were taken to reduce incidences of stock shortages and surpluses. In this month most of the invoices were entered within working hours, which was acknowledged by different managers. There were still errors in coding at branches with new personnel.

May: The delivery of merchandise for the counter was expedited and there was greater availability in the warehouse, training was provided for catalog updates, the stock level of some spare parts groups was increased, and the recurrence of lubricant inventories was increased in order to identify causes of oil shortages. With respect to the tracking charts, there was a setback in compliance due to problems with the coding of claims and observations in the warehouse order due to the arrival of replacement parts for 1.5 months of stock. 
Table 4. Commitment Chart. March-2019.

\begin{tabular}{|c|c|c|c|c|c|c|c|c|}
\hline \multicolumn{7}{|c|}{ CbM - Limautos Spareparts } \\
\hline $\mathbf{N}^{\circ}$ & Date & Commitment & $\begin{array}{c}\text { Requested } \\
\text { by }\end{array}$ & Responsible & $\begin{array}{c}\text { Dead- } \\
\text { line }\end{array}$ & Compliance & Renegociation & Status \\
\hline 1 & $03 / 04 / 19$ & Arrive to work on time & $\begin{array}{c}\text { Assistant } \\
\text { manager }\end{array}$ & Warehouse & $03 / 11 / 19$ & Renegotiated & $18 / 03 / 19$ & Fulfilled \\
\hline 2 & $03 / 04 / 19$ & Conduct oil on date inventory & Analyst & Warehouse & $03 / 11 / 19$ & Renegotiated & $18 / 03 / 19$ & Fulfilled \\
\hline 3 & $03 / 11 / 19$ & $\begin{array}{c}\text { Request training on the Chinese } \\
\text { spare parts catalog }\end{array}$ & $\begin{array}{c}\text { Warehouse } \\
\text { SQ3 }\end{array}$ & Analyst & $03 / 18 / 19$ & Fulfilled & & \\
\hline 4 & $03 / 11 / 19$ & $\begin{array}{c}\text { Send support staff to order shelves } \\
\text { LO }\end{array}$ & $\begin{array}{c}\text { Warehouse } \\
\text { LO }\end{array}$ & Analyst & $03 / 18 / 19$ & Fulfilled & & \\
\hline 5 & $03 / 11 / 19$ & $\begin{array}{c}\text { Enable support for large quotes in } \\
\text { cases of car crash }\end{array}$ & $\begin{array}{c}\text { Warehouse } \\
\text { SQ1 }\end{array}$ & Analyst & $03 / 18 / 19$ & Renegotiated & $25 / 03 / 19$ & Fulfilled \\
\hline 6 & $03 / 18 / 19$ & $\begin{array}{c}\text { Send warehouse photos on Satur- } \\
\text { days for evaluation }\end{array}$ & $\begin{array}{c}\text { Analyst } \\
\text { driver }\end{array}$ & Warehouse & $03 / 25 / 19$ & Fulfilled & & \\
\hline 8 & $03 / 25 / 19$ & $\begin{array}{c}\text { Coordinate on time arrival of route } \\
\text { Warehouse } \\
\text { LO }\end{array}$ & Analyst & $04 / 01 / 19$ & Fulfilled & & \\
\hline
\end{tabular}

Source: Prepared by the author.

Table 5. Commitment Chart. April-2019.

\begin{tabular}{|c|c|c|c|c|c|c|c|c|}
\hline \multicolumn{9}{|c|}{ CbM - Limautos Spareparts } \\
\hline $\mathbf{N}^{\circ}$ & Date & Commitment & $\begin{array}{c}\text { Requested } \\
\text { by }\end{array}$ & Responsible & $\begin{array}{l}\text { Dead- } \\
\text { line }\end{array}$ & Compliance & Renegociation & Status \\
\hline 1 & 04/01/19 & \begin{tabular}{|c|} 
Report invoiced spare parts pending \\
pickup
\end{tabular} & Analyst & Warehouse & $04 / 08 / 19$ & Fulfilled & & \\
\hline 2 & 04/01/19 & $\begin{array}{l}\text { Conduct training in backorder } \\
\text { tracking }\end{array}$ & $\begin{array}{l}\text { Warehouse } \\
\text { SQ2 }\end{array}$ & Analyst & |04/08/19 & Fulfilled & & \\
\hline 3 & 04/08/19 & Enable an additional Kia catalog & $\begin{array}{c}\text { Warehouse } \\
\text { Villarán }\end{array}$ & Analyst & $04 / 15 / 19$ & Not fulfilled & & \\
\hline 4 & 04/08/19 & $\begin{array}{l}\text { Enable an additional Peugeot } \\
\text { catalog }\end{array}$ & $\begin{array}{c}\text { Warehouse } \\
\text { LO }\end{array}$ & Analyst & $04 / 15 / 19$ & Fulfilled & & \\
\hline 5 & $04 / 15 / 19$ & $\begin{array}{l}\text { Conduct cycle counting of type A } \\
\text { items for the workshop }\end{array}$ & Analyst & Warehouse & $04 / 22 / 19$ & Renegotiated & $29 / 04 / 19$ & Fulfilled \\
\hline 6 & $04 / 15 / 19$ & Prioritize spare parts returns on time & $\begin{array}{l}\text { Assistant } \\
\text { manager }\end{array}$ & $\begin{array}{l}\text { Warehouse } \\
\text { SQ2 }\end{array}$ & $04 / 22 / 19$ & Fulfilled & & \\
\hline 7 & $04 / 22 / 19$ & $\begin{array}{l}\text { Send list of damaged covers to } \\
\text { auction }\end{array}$ & $\begin{array}{l}\text { Assistant } \\
\text { manager }\end{array}$ & Analyst & $04 / 29 / 19$ & Renegotiated & $06 / 05 / 19$ & Fulfilled \\
\hline 8 & $04 / 22 / 19$ & $\begin{array}{c}\text { Coordinate arrival date for May } \\
\text { replenishment }\end{array}$ & $\begin{array}{l}\text { Warehouse } \\
\text { SQ2 }\end{array}$ & Analyst & $04 / 29 / 19$ & Fulfilled & & \\
\hline 9 & 04/29/19 & $\begin{array}{l}\text { Process invoice entries before the } \\
\text { closing date }\end{array}$ & Analyst & Warehouse & 05/06/19 & Fulfilled & & \\
\hline
\end{tabular}

Source: Prepared by the author.

Table 6. Commitment Chart. May-2019.

\begin{tabular}{|c|c|c|c|c|c|c|c|c|}
\hline \multicolumn{7}{|c|}{ CbM - Limautos Spareparts } \\
\hline $\mathbf{N}^{\circ}$ & Date & Commitment & $\begin{array}{c}\text { Requested } \\
\text { by }\end{array}$ & Responsible & $\begin{array}{c}\text { Dead- } \\
\text { line }\end{array}$ & Compliance & Renegociation & Status \\
\hline 1 & $05 / 06 / 19$ & $\begin{array}{c}\text { Inventory goods } \\
\text { CUP }>2000 \text { PEN }\end{array}$ & Analyst & Warehouse & $05 / 13 / 19$ & Renegotiated & $20 / 05 / 19$ & Fulfilled \\
\hline 2 & $05 / 06 / 19$ & $\begin{array}{c}\text { Support in delivery of goods to } \\
\text { insurance company }\end{array}$ & $\begin{array}{c}\text { Assistant } \\
\text { manager }\end{array}$ & Warehouse & $05 / 13 / 19$ & Fulfilled & & \\
\hline 3 & $05 / 06 / 19$ & Request training in Peugeot catalog & $\begin{array}{c}\text { Warehouse } \\
\text { SQ1 }\end{array}$ & Analyst & $05 / 13 / 19$ & Fulfilled & & \\
\hline 4 & $05 / 13 / 19$ & $\begin{array}{c}\text { Change of delivery transportation } \\
\text { schedule }\end{array}$ & $\begin{array}{c}\text { Warehouse } \\
\text { Huachipa }\end{array}$ & Analyst & $05 / 13 / 19$ & Fulfilled & & Not \\
\hline 5 & $05 / 13 / 19$ & Evaluate job rotation on Saturdays & $\begin{array}{c}\text { Warehouse } \\
\text { Villarán }\end{array}$ & Analyst & $05 / 20 / 19$ & Renegotiated & $27 / 05 / 19$ \\
\hline 6 & $05 / 13 / 19$ & $\begin{array}{r}\text { Increase stock of brake pads and } \\
\text { discs }\end{array}$ & $\begin{array}{c}\text { Warehouse } \\
\text { SQ3 }\end{array}$ & Analyst & $05 / 20 / 19$ & Fulfilled & & \\
\hline 7 & $05 / 20 / 19$ & $\begin{array}{c}\text { Schedule shipment of non-rotating } \\
\text { goods to Huachipa }\end{array}$ & $\begin{array}{c}\text { Warehouse } \\
\text { LO }\end{array}$ & Analyst & $05 / 20 / 19$ & Fulfilled & & \\
\hline 8 & $05 / 20 / 19$ & $\begin{array}{r}\text { Train personnel for replacement } \\
\text { during vacation }\end{array}$ & $\begin{array}{c}\text { Warehouse } \\
\text { Huachipa }\end{array}$ & Analyst & $05 / 20 / 19$ & Fulfilled & & \\
\hline
\end{tabular}


Table 6. Continuation...

\begin{tabular}{|c|c|c|c|c|c|c|c|c|}
\hline \multicolumn{2}{|c|}{ CbM - Limautos Spareparts } \\
\hline $\mathbf{N}^{\circ}$ & Date & Commitment & $\begin{array}{c}\text { Requested } \\
\text { by }\end{array}$ & Responsible & $\begin{array}{c}\text { Dead- } \\
\text { line }\end{array}$ & Compliance & Renegociation & Status \\
\hline 9 & $05 / 20 / 19$ & $\begin{array}{c}\text { Enable administrative personnel for } \\
\text { support at the reception desk }\end{array}$ & $\begin{array}{c}\text { Warehouse } \\
\text { SQ2 }\end{array}$ & Analyst & $05 / 27 / 19$ & Renegotiated & $03 / 06 / 19$ & Fulfilled \\
\hline 10 & $05 / 27 / 19$ & $\begin{array}{c}\text { Inventory lubricants and make } \\
\text { stocktaking between branches }\end{array}$ & $\begin{array}{c}\text { Assistant } \\
\text { manager }\end{array}$ & Warehouse & $06 / 03 / 19$ & Fulfilled & & \\
\hline 11 & $05 / 27 / 19$ & $\begin{array}{c}\text { Process invoice entries before the } \\
\text { closing date }\end{array}$ & Analyst & Warehouse & $06 / 03 / 19$ & Fulfilled & & \\
\hline
\end{tabular}

Source: Prepared by the author.

Table 7. Commitment Chart. June-2019.

\begin{tabular}{|c|c|c|c|c|c|c|c|c|}
\hline \multicolumn{7}{|c|}{ CbM - Limautos Spareparts } \\
\hline $\mathbf{N}^{\circ}$ & Date & Commitment & $\begin{array}{c}\text { Requested } \\
\text { by }\end{array}$ & Responsible & $\begin{array}{c}\text { Dead- } \\
\text { line }\end{array}$ & Compliance & Renegociation & Status \\
\hline 1 & $06 / 03 / 19$ & $\begin{array}{c}\text { Request the assignment of cleaning } \\
\text { personnel for the warehouse } \\
\text { SQ3 }\end{array}$ & $\begin{array}{c}\text { Warehouse } \\
\text { accounting }\end{array}$ & $\begin{array}{c}\text { Analyst } \\
06 / 10 / 19\end{array}$ & Fulfilled & & \\
\hline 3 & $06 / 03 / 19$ & $\begin{array}{c}\text { Assistant } \\
\text { manager }\end{array}$ & Analyst & $06 / 10 / 19$ & Fulfilled & & \\
\hline 4 & $06 / 10 / 19$ & $\begin{array}{r}\text { Perform evaluation of objectives on } \\
\text { the web platform }\end{array}$ & $\begin{array}{c}\text { Assistant } \\
\text { manager }\end{array}$ & Warehouse & $06 / 10 / 19$ & Fulfilled & & \\
\hline 5 & $06 / 10 / 19$ & $\begin{array}{c}\text { Increase budget for food in rotating } \\
\text { inventories }\end{array}$ & Analyst & $\begin{array}{c}\text { Assistant } \\
\text { manager }\end{array}$ & $06 / 17 / 19$ & Renegotiated & $24 / 06 / 19$ & Fulfilled \\
\hline 6 & $06 / 17 / 19$ & $\begin{array}{c}\text { Request faster quotations from the } \\
\text { importer }\end{array}$ & $\begin{array}{c}\text { Warehouse } \\
\text { SQ1 }\end{array}$ & Analyst & $06 / 17 / 19$ & Fulfilled & & \\
\hline 7 & $06 / 17 / 19$ & $\begin{array}{c}\text { Increase stock of brake tablets and } \\
\text { filters for MIT and FUSO }\end{array}$ & $\begin{array}{c}\text { Warehouse } \\
\text { LO }\end{array}$ & Analyst & $06 / 17 / 19$ & Fulfilled & & \\
\hline 8 & $06 / 24 / 19$ & $\begin{array}{c}\text { Review with Pacifico Seguros the } \\
\text { returns of products }\end{array}$ & $\begin{array}{c}\text { Assistant } \\
\text { manager }\end{array}$ & Warehouse & $06 / 24 / 19$ & Fulfilled & & \\
\hline 9 & $06 / 24 / 19$ & $\begin{array}{c}\text { Coordinate visit with audit prior to } \\
\text { factory visit }\end{array}$ & $\begin{array}{c}\text { Assistant } \\
\text { manager }\end{array}$ & Analyst & $06 / 24 / 19$ & Fulfilled & & \\
\hline 10 & $06 / 24 / 19$ & $\begin{array}{c}\text { Process invoice entries before the } \\
\text { closing date }\end{array}$ & Analyst & Warehouse & $06 / 24 / 19$ & Fulfilled & & \\
\hline
\end{tabular}

Source: Prepared by the author.

Table 8. Commitment Chart. July-2019.

\begin{tabular}{|c|c|c|c|c|c|c|c|c|}
\hline \multicolumn{9}{|c|}{ CbM - Limautos Spareparts } \\
\hline $\mathrm{N}^{\circ}$ & Date & Commitment & $\begin{array}{c}\text { Requested } \\
\text { by }\end{array}$ & Responsible & $\begin{array}{l}\text { Dead- } \\
\text { line }\end{array}$ & Compliance & Renegociation & Status \\
\hline 1 & $07 / 01 / 19$ & $\begin{array}{l}\text { Contact workshops to service sepa- } \\
\text { rate spare parts }\end{array}$ & $\begin{array}{l}\text { Assistant } \\
\text { manager }\end{array}$ & Warehouse & $07 / 08 / 19$ & Fulfilled & & \\
\hline 2 & $07 / 01 / 19$ & $\begin{array}{c}\text { Send support for the return of erro- } \\
\text { neous spare parts }\end{array}$ & $\begin{array}{l}\text { Assistant } \\
\text { manager }\end{array}$ & Analyst & $07 / 08 / 19$ & Fulfilled & & \\
\hline 3 & $07 / 01 / 19$ & $\begin{array}{l}\text { Enable Chery and MG spare parts } \\
\text { catalogs }\end{array}$ & $\begin{array}{l}\text { Warehouse } \\
\text { SQ3 }\end{array}$ & Analyst & $07 / 08 / 19$ & Fulfilled & & \\
\hline 4 & $07 / 08 / 19$ & $\begin{array}{c}\text { Purchase material for packaging - } \\
\text { service to insurance company }\end{array}$ & $\begin{array}{l}\text { Warehouse } \\
\text { SQ2 }\end{array}$ & Analyst & $07 / 08 / 19$ & Fulfilled & & \\
\hline 5 & 07/08/19 & $\begin{array}{l}\text { Send status of destruction of war- } \\
\text { ranty spare parts }\end{array}$ & Analyst & Warehouse & $07 / 15 / 19$ & Fulfilled & & \\
\hline 6 & 07/08/19 & Reduce delay in local purchases & $\begin{array}{c}\text { Warehouse } \\
\text { SQ2 }\end{array}$ & Analyst & $07 / 15 / 19$ & Renegotiated & $22 / 07 / 19$ & Fulfilled \\
\hline 7 & $07 / 15 / 19$ & $\begin{array}{c}\text { Prepare inventory of spare parts to } \\
\text { be picked up (work order) }\end{array}$ & Analyst & Warehouse & $07 / 15 / 19$ & Fulfilled & & \\
\hline 8 & $07 / 15 / 19$ & $\begin{array}{l}\text { Reduce delay in MG and Chery } \\
\text { quotations }\end{array}$ & $\begin{array}{l}\text { Warehouse } \\
\text { SQ3 }\end{array}$ & Analyst & $07 / 15 / 19$ & Renegotiated & $22 / 07 / 19$ & Fulfilled \\
\hline 9 & $07 / 22 / 19$ & $\begin{array}{c}\text { Review spare parts separated by } \\
\text { the system }\end{array}$ & $\begin{array}{l}\text { Warehouse } \\
\text { Villarán }\end{array}$ & Analyst & $07 / 22 / 19$ & Fulfilled & & \\
\hline 10 & 07/29/19 & $\begin{array}{l}\text { Report claims generated by errone- } \\
\text { ous branch lines }\end{array}$ & $\begin{array}{l}\text { Warehouse } \\
\text { LO - SQ1 }\end{array}$ & Analyst & $07 / 22 / 19$ & Fulfilled & & \\
\hline 11 & $07 / 29 / 19$ & $\begin{array}{l}\text { Process invoice entries before the } \\
\text { closing }\end{array}$ & Analyst & Warehouse & $08 / 06 / 19$ & Fulfilled & & \\
\hline
\end{tabular}

Source: Prepared by the author. 
Table 9. Commitment Chart. August-2019.

\begin{tabular}{|c|c|c|c|c|c|c|c|c|}
\hline \multicolumn{9}{|c|}{ CbM - Limautos Spareparts } \\
\hline $\mathbf{N}^{\circ}$ & Date & Commitment & $\begin{array}{l}\text { Requested } \\
\text { by }\end{array}$ & Responsible & $\begin{array}{l}\text { Dead- } \\
\text { line }\end{array}$ & Compliance & Renegociation & Status \\
\hline 1 & $08 / 05 / 19$ & $\begin{array}{l}\text { Update MG, Mitsubishi and Chery } \\
\text { catalogs }\end{array}$ & $\begin{array}{l}\text { Warehouse } \\
\text { LO }\end{array}$ & Analyst & $08 / 12 / 19$ & Fulfilled & & \\
\hline 2 & $08 / 05 / 19$ & Enable MIT and FUSO catalogs & $\begin{array}{l}\text { Warehouse } \\
\text { Huachipa }\end{array}$ & Analyst & $08 / 12 / 19$ & Fulfilled & & \\
\hline 3 & $08 / 12 / 19$ & $\begin{array}{l}\text { Inventory spare parts display } \\
\text { counter }\end{array}$ & Analyst & Warehouse & 08/19/19 & Fulfilled & & \\
\hline 4 & $08 / 12 / 19$ & $\begin{array}{c}\text { Manage cleanliness and order of } \\
\text { accessories area }\end{array}$ & $\begin{array}{l}\text { Assistant } \\
\text { manager }\end{array}$ & Analyst & $08 / 19 / 19$ & Fulfilled & & \\
\hline 5 & $08 / 12 / 19$ & $\begin{array}{l}\text { Excel training for the warehouse } \\
\text { personnel }\end{array}$ & $\begin{array}{l}\text { Assistant } \\
\text { manager }\end{array}$ & Analyst & $08 / 19 / 19$ & Renegotiated & 26/08/19 & Fulfilled \\
\hline 6 & $08 / 19 / 19$ & Train new personnel in ERP & $\begin{array}{l}\text { Warehouse } \\
\text { SQ3 }\end{array}$ & Analyst & $08 / 19 / 19$ & Fulfilled & & \\
\hline 7 & $08 / 19 / 19$ & $\begin{array}{l}\text { Reduce tire and battery service } \\
\text { time }\end{array}$ & $\begin{array}{l}\text { Warehouse } \\
\text { SQ2 }\end{array}$ & Analyst & $08 / 19 / 19$ & Fulfilled & & \\
\hline 8 & $08 / 19 / 19$ & Prepare for KIA factory visit & $\begin{array}{l}\text { Assistant } \\
\text { manager }\end{array}$ & Warehouse & $08 / 19 / 19$ & Fulfilled & & \\
\hline 9 & 08/26/19 & Correct KIA factory visit remarks & Analyst & Warehouse & 09/02/19 & Fulfilled & & \\
\hline 10 & $08 / 26 / 19$ & $\begin{array}{c}\text { Process invoice entries before the } \\
\text { closing }\end{array}$ & Analyst & $\begin{array}{l}\text { Toos Ware- } \\
\text { house }\end{array}$ & $09 / 02 / 19$ & Fulfilled & & \\
\hline
\end{tabular}

Source: Prepared by the author.

June: The personnel had another change related to the punctuality of Saturday inventories, which caused them to be completed on schedule; also, outstanding advances pending of invoicing were identified in order to reduce obsolescence and requests were made to the importer to reduce waiting times (between 1 and 3 days) for quotations of imports. On the other hand, new parameters were negotiated for filter replacements due to the high variability in the last month. In general, the best results in the Commitment-based Management were obtained as there was a reduction of incidents.

July: In order to reduce obsolescence, pending tasks were tracked in the workshop. From another point of view, new catalogs for Chinese spare parts were made available and planning for the destruction of warranty spare parts was started. The importer was asked again to reduce the response time for quotations of imported Chinese spare parts, and steps were taken to return to the factory from the branches with coding errors due to the responsibility of the importer. In addition, there were difficulties in Surquillo3 due to the departure of assigned personnel on vacation and there were errors in the coding of low turnover electrical items.
August: Three catalogs with errors were updated and the personnel retrained in the Huachipa plant. Prior to the factory visit, each warehouse was inspected and order and cleanliness issues were corrected. As part of the annual objectives, training was provided in Excel and SPIGA+ to expedite internal warehouse processes. Coordinated with the purchasing department to expedite quotes for local items. By this time, several locations had already achieved $100 \%$ compliance with the CbM, so the analyst was asked to prioritize the two locations that still have problems.

In general, the team achieved punctuality, on-time compliance of rotating inventories and invoice entry. In addition, priority was given to training and catalog updates, and support was provided to the different branches in overloaded workdays and when replacement personnel were needed for vacations. Finally, the closing of invoice receipts on time was completed, actions that were acknowledged by the Administration Management.

It should be noted that during January and February, the scheme was proposed without actually involving Commitment-based Management, which was considered to be fully initiated as of March 2019.

As explained above, each branch was evaluated in a monthly meeting between the warehouse analyst 
and the spare parts coordinator, which generated the results shown in Table 10.

Growth was observed in the Commitment-based Management compliance indicator, which started with $63 \%$ when the project was not fully implemented and had a value of $88 \%$ by August: a result of the cohesion in the warehouse team.

\section{Contrast de hypotheses: CbM vs. Gross Margin}

Based on Table 8, Table 11 was developed as a summary, where the percentage of CbM compliance during the project was identified:

On the other hand, Table 12 shows the gross margin percentage of the spare parts area from March to August 2019.
It should be noted that since the performance of the workshop channel was under the leadership of the workshop assistant manager, it was decided to use only the value of the counter channel. Also, based on the details of the hypothesis, Table 13 was generated, in which the values for the variables were identified.

Finally, Table 14 was generated, where the correlation coefficient was determined using Excel 2019, Data tab, Analysis section, Data Analysis tool, Regression option.

When reviewing the statistical results, it is observed that the correlation coefficient is 0.52 , which indicates that there is a positive correlation between the dependent and independent variables, so the

Table 10. CbM Rating Chart Results.

\begin{tabular}{|c|c|c|c|c|c|c|c|c|c|c|c|c|c|c|c|c|c|c|c|c|c|c|c|c|c|c|c|c|c|c|c|c|}
\hline & \multicolumn{32}{|c|}{2019} \\
\hline & \multicolumn{4}{|c|}{ January } & \multicolumn{4}{|c|}{ February } & \multicolumn{4}{|c|}{ March } & \multicolumn{4}{|c|}{ April } & \multicolumn{4}{|c|}{ May } & \multicolumn{4}{|c|}{ June } & \multicolumn{4}{|c|}{ July } & \multicolumn{4}{|c|}{ August } \\
\hline & 1 & 2 & 3 & 4 & 1 & 2 & 3 & 4 & 1 & 2 & 3 & 4 & 1 & 2 & 3 & 4 & 1 & 2 & 3 & 4 & 1 & 2 & 3 & 4 & 1 & 2 & 3 & 4 & 1 & 2 & 3 & 4 \\
\hline Surquillo1 & 0 & 1 & 1 & 0 & 0 & 1 & 1 & 0 & 0 & 1 & 1 & 1 & 1 & 1 & 0 & 1 & 1 & 1 & 1 & 0 & 1 & 1 & 1 & 0 & 1 & 1 & 1 & 1 & 1 & 1 & 1 & 1 \\
\hline Surquillo2 & 1 & 0 & 1 & 1 & 0 & 1 & 1 & 0 & 1 & 1 & 1 & 1 & 1 & 1 & 1 & 1 & 0 & 1 & 1 & 1 & 1 & 1 & 1 & 1 & 1 & 1 & 1 & 1 & 1 & 1 & 1 & 1 \\
\hline Surquillo3 & 0 & 1 & 0 & 0 & 1 & 0 & 1 & 0 & 1 & 0 & 0 & 0 & 0 & 1 & 1 & 1 & 0 & 1 & 1 & 0 & 1 & 0 & 1 & 1 & 0 & 1 & 1 & 0 & 1 & 0 & 1 & 0 \\
\hline Villarán & 1 & 1 & 0 & 1 & 1 & 1 & 0 & 1 & 1 & 1 & 0 & 1 & 1 & 1 & 1 & 0 & 1 & 1 & 1 & 0 & 1 & 1 & 1 & 0 & 1 & 1 & 1 & 0 & 1 & 0 & 1 & 1 \\
\hline Los Olivos & 1 & 1 & 1 & 0 & 0 & 1 & 1 & 0 & 1 & 1 & 1 & 0 & 1 & 1 & 1 & 0 & 1 & 1 & 1 & 1 & 1 & 1 & 1 & 1 & 1 & 1 & 1 & 0 & 1 & 1 & 1 & 1 \\
\hline Huachipa & 1 & 1 & 0 & 1 & 1 & 1 & 0 & 1 & 1 & 1 & 1 & 0 & 1 & 1 & 1 & 1 & 1 & 1 & 0 & 1 & 1 & 1 & 1 & 1 & 1 & 1 & 1 & 1 & 1 & 1 & 1 & 1 \\
\hline$\% \mathrm{GpC}$ & & 63 & $\%$ & & & $5 \varepsilon$ & $\%$ & & & 71 & & & & 83 & $\%$ & & & 75 & $\%$ & & & 88 & & & & 83 & & & & & $8 \%$ & \\
\hline
\end{tabular}

Source: Prepared by the author.

Table 11. Summary of the CbM Compliance Chart.

\begin{tabular}{|c|c|}
\hline Month & CbM \\
\hline MAR & $71 \%$ \\
\hline APR & $83 \%$ \\
\hline MAY & $75 \%$ \\
\hline JUN & $75 \%$ \\
\hline JUL & $83 \%$ \\
\hline AUG & $88 \%$ \\
\hline
\end{tabular}

Source: Prepared by the author.

Table 12. Monthly Result of Gross Margin.

\begin{tabular}{|l|l|l|l|l|l|l|l|}
\hline \multicolumn{1}{|c|}{ Gross Margin } & MAR & APR & MAY & JUN & JUL & AUG & YTD \\
\hline General & $33 \%$ & $30 \%$ & $28 \%$ & $27 \%$ & $32 \%$ & $30 \%$ & $\mathbf{3 0} \%$ \\
\hline Canal Mostrador & $25 \%$ & $27 \%$ & $19 \%$ & $25 \%$ & $23 \%$ & $29 \%$ & $\mathbf{2 3 \%}$ \\
\hline Canal Taller & $37 \%$ & $32 \%$ & $31 \%$ & $30 \%$ & $36 \%$ & $33 \%$ & $33 \%$ \\
\hline
\end{tabular}

Source: Prepared by the author. 
Table 13. Identification of Dependent and Independent Variables.

\begin{tabular}{|l|c|c|}
\hline \multicolumn{1}{|c|}{ Month } & Y: Gross Margin & X: CbM \\
\hline MAR & $25 \%$ & $71 \%$ \\
\hline APR & $27 \%$ & $83 \%$ \\
\hline MAY & $19 \%$ & $75 \%$ \\
\hline JUN & $25 \%$ & $75 \%$ \\
\hline JUL & $23 \%$ & $83 \%$ \\
\hline AUG & $29 \%$ & $88 \%$ \\
\hline
\end{tabular}

Source: Prepared by the author.

Table 14. Regression Statistics.

\begin{tabular}{|l|c|}
\hline \multicolumn{2}{|c|}{ Regression Statistics } \\
\hline Coefficient of multiple correlation & 0.52467218 \\
\hline Coefficient of determination $\mathrm{R}^{\wedge} 2$ & 0.2752809 \\
\hline Adjusted $\mathrm{R}^{\wedge} 2$ & 0.09410112 \\
\hline Standard error & 0.03278719 \\
\hline Observations & 6 \\
\hline
\end{tabular}

Source: Prepared by the author.

hypothesis stated is considered valid. However, when analyzing the coefficient of determination $\mathrm{R} 2$, a value of 0.275 is observed, which means that the model slightly explains the variability of the response data as a function of its mean, so the data may be far from the regression graph.

Also, it is detailed in the analysis provided by Excel 2019, where the regression equation is as follows:

$$
Y=0.025+0.28 X
$$

This equation indicates that there is a positive slope, which reinforces the affirmation of the positive correlation between the variable "compliance level of Commitment-based Management in the spare parts warehouse area" and the variable "gross margin of the spare parts sub-management with respect to total sales".

\section{DISCUSSION}

With respect to the previous point, it is confirmed that the higher the compliance with Commitment-based Management, the higher the gross margin of the spare parts management, according to the success cases previously presented in the implementation of Commitment-based Management in the companies Repsol and Divemotor. Divemotor, which belongs to the same business sector, managed to increase the average monthly workshop and counter sales while increasing its gross margin; just like the company analyzed in this study.
On the other hand, when reviewing the CbM compliance chart, there is an increase in compliance with CbM since March, the date on which the CbM training for the area in question was validated. In addition, there is a drop in compliance during some months, which suggests the presence of internal factors that generated a delay in the project during some months.

Likewise, after the analysis, a pattern for the development of CbM among collaborators and managers was observed, which leaves room to evaluate the application of this tool in other organizations with a position structure similar to the one proposed in this study and with operational personnel with high school or technical studies, for which the following steps are recommended:

1. Identify the critical factors that require monitoring.

2. Request authorization from the corresponding management, supporting the necessity of the project for an improvement in gross margin.

3. Start the initial $\mathrm{CbM}$ training meeting to develop bonds among collaborators. Also, prioritize the win-win mentality as the initial axis of the commitments.

4. Generate a commitment chart with the indicators of the identified factors.

5. Appoint personnel for monitoring and elaborate a meeting schedule. 
6. Initiate meetings according to the proposed methodology. In the meetings, the majority of the team should participate in order to increase the confidence of the personnel. Commitments must be achievable and must be developed with responsibility on both sides.

7. After the first six months of the project, carry out the first evaluation of the gross margin to recognize the results and make the necessary adjustments to provide continuity to the project.

Additionally, it is proposed to start in an End-to-End area to reduce the presence of external factors that may complicate the fulfillment of commitments. Likewise, it is proposed to extrapolate this tool to other companies that have a structure focused on sales and do not have a process and quality area, such as lubrication stations, mechanical workshops, spare parts distributors, accessory dealers, among other companies in the automotive industry.

\section{CONCLUSIONS AND RECOMMENDATIONS}

This study concludes that there is a positive correlation between the level of compliance with Commitment-based Management and the gross margin of the spare parts sub-management focused on the counter, which is supported by the growth of the margin during the execution of the proposed project.

Commitment-based Management serves as a tool to facilitate teamwork, to align efforts in search of common objectives, to provide empowerment to operating personnel and to collaborate with the development of the new generations that are entering into the labor market and have different motivations and goals.

It is recommended that the model presented is applied over a longer period of time to obtain more information and numerical data that will allow a complete analysis of the variability of the data with respect to the regression indicated. Likewise, the methodology presented should be applied to the workshop sub-management to obtain a complete picture of the influence of Commitment-based Management in the spare parts sub-management of the company in study.

\section{ACKNOWLEDGMENT}

- Universidad Nacional Mayor de San Marcos

- Limautos spare parts sub-management

\section{REFERENCES}

[1] Asociación Automotriz del Perú. (2019). AAP: los efectos de un parque automotor escaso y antiguo. Retrieved from https://aap.org.pe/aaplos-efectos-de-un-parque-automotor-escaso-yantiguo-2/

[2] Asociación Automotriz del Perú. (2020). Importación de suministros 2018. Retrieved from https://aap.org.pe/estadisticas/ importacion_suministros/importacion-desuministros-2019/

[3] Dann, J. (2009). Manage by commitments, not hierarchies by Donald Sull. CBS NEWS. Retrieved from https://www.cbsnews.com/ news/donald-sull-manage-by-commitmentsnot-hierarchies/

[4] D. S. N. ${ }^{\circ}$ 095-2018-EF. Modifican el Literal A del Nuevo Apéndice IV del Texto Único Ordenado de la Ley del Impuesto General a las Ventas e Impuesto Selectivo al Consumo. Diario Oficial El Peruano (miércoles 9 de mayo de 2018).

[5] D. S. N. ${ }^{\circ} 181-2019-E F$. Decreto Supremo que modifica el Impuesto Selectivo al Consumo aplicable a los bienes del Nuevo Apéndice IV del TUO de la Ley del Impuesto General a las Ventas e Impuesto Selectivo al Consumo y el Reglamento de la Ley del Impuesto a la Renta. Diario Oficial El Peruano (sábado 15 de junio de 2019).

[6] Divemotor. (2017). La Empresa. Retrieved from https://www.divemotor.com/empresa/

[7] Dougherty, J. (2012). To get a commitment, make a commitment. Harvard Business Review. Retrieved from https://hbr.org/2012/12/to-geta-commitment-make-a-com

[8] Gestión. (2015). El 87\% de empresas considera que la falta de compromiso laboral es su principal problema. Retrieved from https://gestion.pe/tendencias/managementempleo/87-empresas-considera-faltacompromiso-laboral-principal-problema105592-noticia/?ref=gesr

[9] Goldsmith, M. (2008). How to increase Employee Commitment. Retrieved from https:// hbr.org/2008/01/how-to-increase-employeecommi

[10] INEI. (2020). Actividad comercial aumentó $3,47 \%$ en noviembre de 2019. Indicadores Económicos, Boletín Estadístico 02. Retrieved from https://www.inei.gob.pe/ 
media/MenuRecursivo/boletines/boletinquincenal-02_2.pdf

[11] Limautos Automotriz del Perú S.A.C. (n.d.). Misión. Retrieved from https://www.limautos. pe/nosotros

[12] Negri, A. (2019). 2020: Una meta en común. Asociación Automotriz del Perú, Boletín 125.

[13] Newstrom, J.W. (2011). Comportamiento humano en el trabajo. México D.F., Mexico: McGraw-Hill.

[14] Ramos, L., \& Albitres, R. (2010). Sistema de gestión para resultados en el Perú. (Tesis de maestría). Universidad Nacional de Ingeniería, Lima.

[15] Real Academia Española (n.d.). Compromiso. En Diccionario de la lengua española. Retrieved from http://dle.rae.es/srv/ search? $\mathrm{m}=30 \& \mathrm{w}=$ compromiso

[16] Repsol. (2013). Informes 2013. Equipo Repsol. Retención del Talento.

[17] Sull, D. (2003). Managing by commitments. Harvard Business Review. Retrieved from https:// hbr.org/2003/06/managing-by-commitments
[18] Sull, D.N., \& Spinosa, C. (2005). Using commitments to manage across units. MIT Sloan Management Review, 47 (1), 73-81. Retrieved from https://www.researchgate.net/ publication/40968864

[19] Stein, G., \& Pin, J.R. (2009). Como dirigir a las nuevas generaciones de profesionales: motivaciones y valores de la generación $\mathrm{Y}$. Harvard Deusto Business Review, (178). Retrieved from https://www.harvard-deusto. com/como-dirigir-a-las-nuevas-generacionesde-profesionales-motivaciones-y-valores-dela-generacion-y

[20] Viato, R. (January 12, 2014). Poderosa herramienta. Revista $D$. Retrieved from https:// www.prensalibre.com/revista-d/coachingliderazgo-recursos-humanos-gestion-detiempo-desarrollo-profesional-0-1062493984/ 